\title{
Muon $g-2$ in an alternative quasi-Yukawa unification with a less fine-tuned seesaw mechanism
}

\author{
Zafer Altın, ${ }^{1, *}$ Özer Özdal, ${ }^{2, \dagger}$ and Cem Salih Ün ${ }^{1, \ddagger}$ \\ ${ }^{1}$ Department of Physics, Uluda ğ University, TR16059 Bursa, Turkey \\ ${ }^{2}$ Department of Physics, Concordia University, \\ 7141 Sherbrooke Street West, Montreal, Quebec H4B 1R6, Canada
}

(Received 23 June 2017; published 6 March 2018)

\begin{abstract}
We explore the low-scale implications of the Pati-Salam Model including the $\mathrm{TeV}$ scale right-handed neutrinos interacting and mixing with the MSSM fields through the inverse seesaw (IS) mechanism in light of the muon anomalous magnetic moment (muon $g-2$ ) resolution and highlight the solutions which are compatible with the quasi-Yukawa unification condition (QYU). We find that the presence of the righthanded neutrinos causes heavy smuons as $m_{\tilde{\mu}} \gtrsim 800 \mathrm{GeV}$ in order to avoid tachyonic staus at the low scale. On the other hand, the sneutrinos can be as light as about $100 \mathrm{GeV}$, and along with the light charginos of mass $\lesssim 400 \mathrm{GeV}$, they can yield such large contributions to muon $g-2$ that the discrepancy between the experiment and the theory can be resolved. These solutions also require $m_{\tilde{\chi}_{1}^{ \pm}} \lesssim 400 \mathrm{GeV}$ and $m_{\tilde{\chi}_{1}^{0}} \lesssim 200$. We also discuss such light chargino and neutralino along with the light stau $\left(m_{\tilde{\tau}} \gtrsim 200 \mathrm{GeV}\right)$ in the light of current LHC results. Besides, the gluino mass lies in a range $\sim[2.5-3.5] \mathrm{TeV}$, which is tested in near future experiments. In addition, the model predicts relatively light Higgsinos $(\mu \lesssim 700 \mathrm{GeV})$; hence, the second chargino mass is also light enough $(\lesssim 700 \mathrm{GeV})$ to contribute to muon $g-2$. Light Higgsinos also yield less fine-tuning at the electroweak scale, and the regions compatible with muon $g-2$ restrict $\Delta_{\mathrm{EW}} \lesssim 100$ strictly, and this region also satisfies the QYU condition. In addition, the ratios among the Yukawa couplings should be $1.8 \lesssim y_{t} / y_{b} \lesssim 2.6, y_{\tau} / y_{b} \sim 1.3$ to yield correct fermion masses. Even though the righthanded neutrino Yukawa coupling can be varied freely, the solutions bound its range to $0.8 \lesssim y_{\nu} / y_{b} \lesssim 1.7$.
\end{abstract}

DOI: 10.1103/PhysRevD.97.055007

\section{INTRODUCTION}

Supersymmetry (SUSY) is one of the leading candidates for physics beyond the standard model (SM). Resolving the gauge hierarchy problem the Higgs boson mass is not too much sensitive to the ultraviolet scale. In addition, the minimal supersymmetric version of the SM (MSSM) nicely unifies the three gauge couplings of the SM, and hence, one can identify the unification scale as $M_{\mathrm{GUT}} \sim 2 \times 10^{16} \mathrm{GeV}$. In this context, SUSY models can study the high energy origins of physics by connecting $M_{\mathrm{GUT}}$ to the electroweak scale through the renormalization group equations (RGEs). Supersymmetric grand unified theories (SUSY GUTs) allow one to build high scale models, and these models can significantly reduce the number of free parameters in

\footnotetext{
*501407009@ogr.uludag.edu.tr

†ozer.ozdal@concordia.ca

¥cemsalihun@uludag.edu.tr
}

Published by the American Physical Society under the terms of the Creative Commons Attribution 4.0 International license. Further distribution of this work must maintain attribution to the author(s) and the published article's title, journal citation, and DOI. Funded by SCOAP . comparison to the low-scale MSSM models with free parameters more than a hundred. In this approach, minimal SUSY models have been built such as constrained MSSM (CMSSM) and nonuniversal Higgs models (NUHM), and their phenomenological implications have been excessively explored. These minimal models have been built with the inspiration from $S O(10)$ GUTs. These GUT models do not only unify the gauge couplings, but the matter fields are also unified into a single representation. Since the spinor representation of $S O(10)$ is 16-dimensional, there is still an empty spot for the rigth-handed neutrino after placing all the matter particles. In this sense, $S O(10)$ GUTs provide a natural framework to implement the SeeSaw mechanisms through which the neutrinos mix each other and receive nonzero masses favored by the current experiments [1].

Besides the gauge coupling and matter fields unification, another interesting feature of the $S O(10)$ GUTs is the unification of the Yukawa couplings (YU) [2]. This unification scheme is also shared by the high scale models, which preserve the symmetry under transformations belonging to the $S O(10)$ 's maximal subgroup, which is the Pati-Salam gauge group $G_{\mathrm{PS}}=S U(4)_{C} \times S U(2)_{L} \times$ $S U(2)_{R}$ (hereafter 4-2-2 for short) [3]. Even though it does not provide a GUT model $\left(g_{4} \neq g_{L} \neq g_{R}\right.$ in principle), 
if it breaks into the MSSM gauge group at a scale near by $M_{\mathrm{GUT}}$, the gauge couplings receive negligible threshold corrections; hence, the gauge coupling unification can be maintained in $4-2-2$ as well. In addition, imposing leftright (LR) symmetry requires $g_{L}=g_{R} \equiv g_{2}$ and, consequently, $M_{L}=M_{R} \equiv M_{2}$. The hypercharge generator can be expressed in terms of the diagonal generators in $G_{\mathrm{PS}}$ as

$$
Y=\sqrt{\frac{3}{5}} I_{3 R}+\sqrt{\frac{2}{5}}(B-L),
$$

where $I_{3 R}$ and $B-L$ are diagonal generators of $S U(2)_{R}$ and $S U(4)_{C}$ symmetry groups, respectively. This relation for the hypercharge generator also yields nonuniversal gaugino mass terms for the MSSM gaugino fields as

$$
M_{1}=\frac{3}{5} M_{2}+\frac{2}{5} M_{3},
$$

with $M_{1}, M_{2}$, and $M_{3}$ being soft supersymmetry breaking (SSB) mass terms for the MSSM gauginos associated with the $U(1)_{Y}, S U(2)_{L}$, and $S U(3)_{C}$ gauge groups, respectively.

Despite being imposed at the GUT scale, the YU condition provides an exclusive impact on the low-scale phenomenology. This impact is mostly based on the fact that the bottom Yukawa coupling needs to receive the largest negative threshold corrections at the low scale in order to yield consistent masses for the top and bottom quarks [4]. On the other hand, even though it is a very effective condition from $M_{\mathrm{GUT}}$ to shape the low-scale parameter space, YU fails to yield consistent fermion masses for the first two families, since it predicts $N=U \propto D=L$, where $N, U, D, L$ are Dirac mass matrices for right-handed neutrinos, up and down quarks, and charged leptons, respectively. In addition, the proportionality between the up and down quarks results in vanishing flavor (Cabibbo-Kobayashi-Maskawa) mixing [5]. Also the mass relations resulted from YU $m_{c}^{0} / m_{t}^{0}=$ $m_{s}^{0} / m_{b}^{0}$ are clearly contradicted with the experimental results, where the superscript zero refers to the parameters evaluated at $M_{\mathrm{GUT}}$. In order to correct these mass relations of the fermions, one can add new vectorlike matter multiplets, which can mix with the fermions [6]. Another approach is to extend the content with new Higgs fields from another representations [7]. In this case, one can assume that the extra Higgs fields negligibly interact with the third family matter fields, and the MSSM Higgs doublets reside solely in 10-plet of $S O(10)$ to maintain YU for the third family fermions, while the mass relations for the first two family fermions are corrected with these extra Higgs fields [8].

On the other hand, if we do not follow the assumptions mentioned above, the two approaches break YU. In this case, if we restrict the deviations in the Yukawa couplings of the third family up to, say 20\%; then, this modified unification scheme is called QYU [9]. Even though the deviation is restricted to small amounts, QYU yields drastically different phenomenology at the low scale. For instance, $4-2-2$ is the only model, as to our knowledge, which yields light gluino $\left(m_{\tilde{g}} \lesssim 1 \mathrm{TeV}\right)$ to be next to lightest supersymmetric particle (NLSP), when YU is imposed at the GUT scale [10]. Relaxing it to $b-\tau$ YU allows stop NLSP solutions in addition to gluino [11]. On the other hand, QYU in 4-2-2 allows a variety of NLSP species, while stop and gluino NLSP solutions are not compatible with QYU [12]. In addition, the parameter space compatible with YU yields large fine-tuning. While QYU can be realized with acceptable fine-tuning [12].

Based on the discussion above, YU provides a strict framework, in which the representations from a possible GUT gauge group is rather required to be minimal, since the MSSM Higgs fields are allowed to reside in $S O(10)$ 's $10-$ plet. Even though the framework can be extended in the QYU case, it is still minimalistic since only one extra representation for the Higgs fields (with those from $(15,1,3)$ [9]) is allowed. However, if the framework is extended to include other possible Higgs representations, the MSSM Higgs fields become linear superpositions of those from these representations, and the Yukawa couplings can receive different contributions depending on the vertices between the relevant matter and Higgs fields [13]. In addition to the extra Higgs fields, the presence of higher dimensional operators also contribute to the Yukawa couplings such that the top quark Yukawa coupling can receive a significant correction from such operators [14], and its deviation from YU cannot be restricted within $t-b-\tau$ QYU. In this context, the unification scheme can be identified as $b-\tau$ QYU [15].

The discussion followed so far does not consider the righthanded neutrinos. If LR symmetry is imposed in $4-2-2$, it requires the existence of the right-handed neutrinos, which actively participate in interactions through $S U(2)_{R}$ gauge group. Usually the effects from the right-handed neutrinos can be neglected safely due to the smallness of neutrino masses established by the experiments [1], which stringently restricts the neutrino Yukawa coupling as $y_{\nu} \lesssim 10^{-7}$ [16]. On the other hand, this result does not hold when the inverse SeeSaw (IS) mechanism is implemented, in which a large neutrino Yukawa coupling $\left(y_{\nu} \sim y_{t}\right)$ can still be consistent with the smallness of neutrino masses [17]. With the presence of the right-handed neutrinos with a large Yukawa coupling, the unification scheme discussed above should be modified to include the right-handed neutrinos. In this case YU should be imposed as $y_{t}=y_{b}=y_{\tau}=y_{\nu} \equiv y$ at $M_{\mathrm{GUT}}$. In the case of QYU, the deviations in $y_{\nu}$ and $y_{t}$ from YU are related. In this context, the models predicting $b-\tau$ QYU at $M_{\mathrm{GUT}}$ should also be compatible with $t-\nu$ QYU simultaneously. Following Refs. [9,12], the deviations in Yukawa couplings can be formulated as

$$
\begin{aligned}
& y_{b}: y_{\tau}=\left|1-C_{b \tau}\right|:\left|1+3 C_{b \tau}\right| \\
& y_{t}: y_{\nu}=\left|1+C_{t \nu}\right|:\left|1-3 C_{t \nu}\right|,
\end{aligned}
$$


where $C_{b \tau}$ and $C_{t \nu}$ measure the deviation in Yukawa couplings from YU. Note that since $\mathrm{YU}$ is broken first by the higher order operators as $y_{t}=y_{\nu}$ and $y_{b}=y_{\tau}, C_{b \tau}$ and $C_{t \nu}$ does not have to be related to each other.

Previous studies of QYU (see for instance Refs. [9,12]) have revealed that the general QYU scenarios are mostly compatible in the regions with large $\tan \beta$. Such regions, depending on the mass spectrum of the supersymmetric particles, can also yield large SUSY contributions to muon $g-2$. The SM predictions exhibit about $3 \sigma$ deviation from the experimental results, and this situation can be expressed as [18]

$$
\Delta a_{\mu} \equiv a_{\mu}^{\mathrm{exp}}-a_{\mu}^{\mathrm{SM}}=(28.7 \pm 8.0) \times 10^{-10}(1 \sigma) .
$$

This discrepancy has been survived even after highly accurate calculations over the SM predictions were performed [19]; therefore, it can be interpreted as the effect of the new physics beyond the SM. The gap between the SM predictions and the experimental results can be filled by imposing nonuniversality at the GUT scale in the scalar and/ or gaugino sector in MSSM without extending the particle content. However, in the case of nonuniversal gauginos, the muon $g-2$ solution favors the models in which the gaugino mass parameters have different signs [20]. In our work, we will explore the low-scale implications of $4-2-2$ including the $\mathrm{TeV}$ scale right-handed neutrinos, which interact and mix with the MSSM fields through the IS mechanism in the light of muon $g-2$ resolution, and highlight the solutions which are compatible with the QYU condition given in Eq. (3). The rest of the paper is organized as follows: We will briefly discuss the effect of the presence of the right-handed neutrinos on muon $g-2$ along with the sparticle mass spectrum in Sec. II. We describe our scanning procedure and the experimental constraints employed in our data generation and analyses are summarized in Sec. III. Then, we first present our results for muon $g-2$ and the mass spectrum for the relevant particles in Sec. IV. Section V discusses the supersymmetric mass spectrum in more details. Section VI discusses muon $g-2$ resolution in respect of the finetuning, which is required to have correct electroweak symmetry breaking scale. We also discuss the light chargino and neutralino solutions and teh current LHC results in Section VII. Finally, we summarize and conclude in Sec. VIII.

\section{MUON $g-2$ IN MSSM WITH INVERSE SEESAW}

In this section, we discuss the SUSY contributions to muon $g-2$ in MSSM, when the right-handed neutrinos are present and they mix through the IS mechanism. The IS mechanism enlarges the MSSM superpotential as follows [21]

$$
\begin{aligned}
W_{\mathrm{IS}}= & W_{\mathrm{MSSM}}+Y_{\nu}^{i j} L_{i} H_{u} N_{j}^{c}+Y_{N}^{i j} S N_{i}^{c} N_{j}^{c} \\
& +M_{R} S N^{c}+\mu_{S} S S
\end{aligned}
$$

where $W_{\text {MSSM }}$ is the usual MSSM superpotential, while the other terms are arised from the IS mechanism. $N^{c}$ and $S$ are the MSSM gauge singlet fields, and the former is identified as the right-handed neutrino field. The MSSM gauge singlet field denoted by $S$ can be involved in many extensions such as Next to MSSM (NMSSM) [22], $B-L$ extended MSSM [17] and so on. Even though it is not related to any symmetry in the MSSM framework, the right-handed neutrino masses are strongly related to vacuum expectation value (VEV) of $S$. Indeed, with its VEV, the solutions consistent with the tiny neutrino masses can be realized even when $M_{R} \sim 1 \mathrm{TeV}$ and $\mathcal{O}\left(Y_{\nu}\right) \sim 1$ [21]. Thus, the advantage of the IS mechanism is to realize $\mathrm{TeV}$ scale right-handed neutrinos with a significant Yukawa coupling to the Higgs boson. In this context, the right-handed neutrinos significantly interfere in the low-scale phenomenology. At the first glance, they interact with $H_{u}$ as the stop does, and hence the contributions to the Higgs boson mass can relax the heavy stop requirement to satisfy the $125 \mathrm{GeV}$ Higgs boson constraint. In such solutions, also the large $A$ term in the stop sector may not be required, and hence one can obtain acceptable fine-tuning to realize the correct electroweak symmetry breaking scale. In this case, it is peculiar that the Higgsinos are sufficiently light $(\mu \lesssim 1 \mathrm{TeV})$ that they can also contribute to the muon anomalous magnetic moment. We present two diagrams which illustrate the righthanded sneutrino contributions to muon $g-2$. Note that these contributions are additional to the usual MSSM contributions [23]. The right-handed sneutrino contributions comes along with the charged sector. The behavior of the SUSY contributions can be understood by calculating these diagrams with the mass insertion method, which is represented with dots in the diagrams. The approximate contributions can be obtained as follows [24]:

$$
\begin{array}{r}
\left(\Delta a_{\mu}\right)_{C 1} \approx \frac{m_{\mu}^{2} \mu^{2} \cot \beta}{m_{\tilde{N}}^{2}-m_{\tilde{\nu}}^{2}}\left[\frac{f_{\chi}\left(\mu^{2} / m_{\tilde{N}}^{2}\right)}{m_{\tilde{N}}^{2}}-\frac{f_{\chi}\left(\mu^{2} / m_{\tilde{\nu}}^{2}\right)}{m_{\tilde{\nu}}^{2}}\right] \\
\left(\Delta a_{\mu}\right)_{C 2} \approx \frac{m_{\mu}^{2} M_{2} \mu \tan \beta}{m_{\tilde{N}}^{2}}\left[\frac{f_{\chi}\left(M_{2}^{2} / m_{\tilde{N}}^{2}\right)-f_{\chi}\left(\mu^{2} / m_{\tilde{N}}^{2}\right)}{M_{2}^{2}-\mu^{2}}\right]
\end{array}
$$

The $\tan \beta$ dependence of muon $g-2$ contributions can be seen from these equations. Note that there are other terms, which do not depend on $\tan \beta$, but these terms are rather negligible, unless the supersymmetric particles are so light that they are excluded by the current mass bounds. The two diagrams shown in Fig. 1 exhibit different behavior in respect of $\tan \beta$. The first diagram is expected to be effective, when $\tan \beta$ is rather small. Recall that if the charged sleptons ran in the loop, the contributions would be enhanced with $\tan \beta$. The suppression in the sneutrino case is because the sneutrinos interact with $H_{u}$, while charged sleptons interact with $H_{d}$. On the other hand, the contributions represented in the second diagram exhibit an enhancement as $\tan \beta$ increases. In these processes, the $\tan \beta$ enhancement arises from the mixing between two 

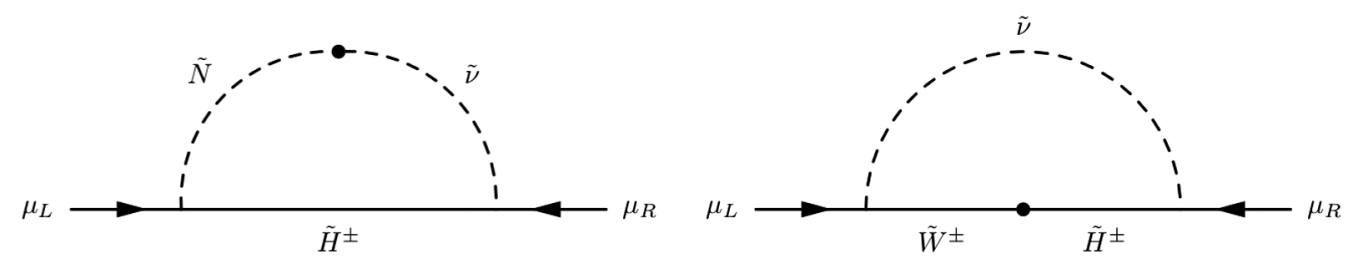

FIG. 1. The SUSY contributions to muon $g-2$ involving with the sneutrinos and charginos.

charginos; i.e. the wino and Higgsino. One can expect that the second diagram illustrates the dominant processes in the SUSY contributions to muon $g-2$.

We should note here that even though the second diagram is already included in the usual MSSM framework, it is not enough to solve the muon $g-2$ discrepancy. On the other hand, supplemented with the IS mechanism, the coupling between the sneutrino and Higgsino can be significantly enhanced, and it can even provide the dominant contributions in the regions with large $\tan \beta$. In addition, the effect of the right-handed neutrinos on the other particles relevant to the muon $g-2$ problem can be understand by considering the following RGEs, which run the parameters from $M_{\mathrm{GUT}}$ to the low scale

$$
\begin{aligned}
\frac{d m_{\tilde{L}}^{2}}{d t}= & \left(\frac{d m_{\tilde{L}}^{2}}{d t}\right)_{M S S M}-2 m_{H_{u}}^{2} Y_{\nu}^{\dagger} Y_{\nu}-2 T_{\nu}^{\dagger} T_{\nu} \\
& -2 m_{\tilde{l}}^{2} Y_{\nu}^{\dagger} Y_{\nu}-2 Y_{\nu}^{\dagger} m_{\tilde{\nu}}^{2} Y_{\nu} \\
\frac{d m_{\tilde{e}}^{2}}{d t}= & \left(\frac{d m_{\tilde{e}}^{2}}{d t}\right)_{M S S M} \\
\frac{d m_{\tilde{N}}^{2}}{d t}= & -2\left(2 m_{H_{u}}^{2} Y_{\nu} Y_{\nu}^{\dagger}+2 T_{\nu} T_{\nu}^{\dagger}+2 Y_{\nu} m_{\tilde{l}}^{2} Y_{\nu}^{\dagger}+2 m_{\tilde{\nu}}^{2} Y_{\nu} Y_{\nu}^{\dagger}\right) \\
\frac{d m_{H_{u}}^{2}}{d t}= & \left(\frac{d m_{H_{u}}^{2}}{d t}\right)_{M S S M}-2 m_{H_{u}}^{2} \operatorname{Tr}\left(Y_{\nu} Y_{\nu}^{\dagger}\right) \\
& -2 \operatorname{Tr}\left(T_{\nu}^{*} T_{\nu}^{T}\right)-2 \operatorname{Tr}\left(m_{\tilde{l}}^{2} Y_{\nu}^{\dagger} Y_{\nu}\right)-2 \operatorname{Tr}\left(m_{\tilde{\nu}}^{2} Y_{\nu} Y_{\nu}^{\dagger}\right) \\
\frac{d \mu}{d t}= & \left(\frac{d \mu}{d t}\right)_{M S S M}-\mu \operatorname{Tr}\left(Y_{\nu} Y_{\nu}^{\dagger}\right)
\end{aligned}
$$

where we have used the usual notation for the MSSM fields. In addition, $\tilde{N}$ denotes the right-handed sneutrino field. The first terms in the equations with the subscript MSSM represent the RGEs for these parameters within the MSSM framework without the right-handed neutrino. The other terms are relevant to the presence of the right-handed neutrinos. As is seen from the RGEs given above, the neutrino Yukawa couplings, $Y_{\nu}$, and its trilinear interaction term $T_{\nu}$ are effective in lowering the SSB masses of the charged sleptons, and as a result they could be much lighter at the low scale than those in the usual MSSM without the right-handed neutrinos. If the texture of the neutrino Yukawa couplings are similar to the up-type quarks Yukawa couplings $\left(Y_{\nu} \sim Y_{u}\right)$ [17], then neutrino Yukawa couplings and trilinear interaction term can result in tachyonic states $\left(m_{L, e, N}^{2}<0\right)$ especially for staus. In this context, the smuon masses can be found slightly heavier in order to avoid tachyonic stau mass eigenstates [25], when $m_{\tilde{\mu}}=m_{\tilde{\tau}}=m_{0}$ at $M_{\mathrm{GUT}}$. However, the SUSY contributions to muon $g-2$ from smuon-neutralino loop can be suppressed, if $m_{\tilde{\mu}} \gtrsim 800$ [20].

Similar discussion holds for the right-handed sneutrinos. Its SSB mass parameter is determined with the common mass scale for the scalars, $m_{0}$; and hence, $m_{0}$ cannot be lower than certain scales not to have tachyonic sneutrinos at the low scale. The RGE for $m_{H_{u}}$ reveals an interesting feature for the IS mechanism that $Y_{\nu}$ and $T_{\nu}$ lower its value from $M_{\mathrm{GUT}}$ to the low scale as $Y_{t}$ gives the same impact in MSSM. The electroweak symmetry breaking requires $m_{H_{u}}^{2}<0$, and MSSM can have only stops to have negative $m_{H_{u}}^{2}$ at the low scale. This fact leads to heavy stops and/or large mixing between left and right-handed stops in the MSSM. On the other hand, when the IS mechanism is implemented in the MSSM framework, the sneutrinos, together with the stops, yield $m_{H_{u}}^{2}<0$, which loose the pressure on the stop sector.

Before concluding this section, the last RGE for the $\mu$ term in Eq. (8) is also interesting in the naturalness point of view. As shown in previous studies [26], the required finetuning at the electroweak scale is mostly determined by $\mu$. Its RGE in the case with the IS mechanism shows that the $\mu$ term is lowered further than that in the usual MSSM by the neutrino Yukawa couplings and trilinear scalar interaction terms. Hence, one can expect that the MSSM with IS can yield significantly low fine-tuned solutions at the low scale.

\section{SCANNING PROCEDURE AND EXPERIMENTAL CONSTRAINTS}

In scanning the fundamental parameter space, we have employed SPheno 3.3.3 package [27] obtained with SARAH 4.6.0 [28]. This package evolves the weak scale values of gauge and Yukawa couplings to $M_{\mathrm{GUT}}$ via the MSSM RGEs, which are modified to include the IS mechanism. $M_{\mathrm{GUT}}$ is dynamically determined with the gauge coupling unification condition. Note that we do not strictly enforce the unification condition at $M_{\mathrm{GUT}}$, since a few percent deviation from the unification can be assigned to unknown GUT-scale threshold corrections [29], which modify the unification condition as $g_{1}=g_{2} \approx g_{3}$. With the boundary conditions given at $M_{\mathrm{GUT}}$, all the SSB parameters 
along with the gauge and Yukawa couplings are evolved back to the weak scale.

We have performed random scans over the following parameter space

$$
\begin{aligned}
0 & \leq m_{0}, \quad m_{H_{d}}, \quad m_{H_{u}} \leq 5 \mathrm{TeV} \\
-5 & \leq M_{2} \leq 0 \mathrm{TeV} \\
0 & \leq M_{3} \leq 5 \mathrm{TeV} \\
-3 & \leq A_{0} / m_{0} \leq 3 \\
35 & \leq \tan \beta \leq 60 \\
\mu & <0, \quad m_{t}=173.3 \mathrm{GeV}
\end{aligned}
$$

where $m_{0}$ symbolizes the universal SSB mass term for the matter scalars, while $m_{H_{d}}$ and $m_{H_{u}}$ denote the SSB mass terms for the MSSM Higgs doublets. $M_{2}$ and $M_{3}$ stand for the gauginos associated with the $S U(2)_{L}$ and $S U(3)_{C}$, respectively. The SSB mass term, $M_{1}$ for the $U(1)_{Y}$ gaugino is determined in terms of $M_{2}$ and $M_{3}$ as given in Eq. (2). $A_{0}$ is the SSB trilinear coupling, and $\tan \beta$ is the ratio of VEVs of the MSSM Higgs doublets. The value of the $\mu$ term is determined by the radiative electroweak symmetry breaking (REWSB) condition but not its sign; thus, its sign is one of the free parameter in MSSM and it is set negative in our scans. In addition, we have employed the current central value for the top quark mass as $m_{t}=173.3 \mathrm{GeV}$ [30]. Note that the sparticle spectrum is not too sensitive in one or two sigma variation in the top quark mass [31], but it can shift the Higgs boson mass by $1-2 \mathrm{GeV}$ [32]. In addition to these free parameters, the experiments do not provide any value for the neutrino's Yukawa coupling at the low scale, in contrast to those associated with the charged leptons. Hence, they need to be provided as an input at the low scale. In our scans, we vary Yukawa coupling $Y_{\nu}$ within perturbative level. We also fix $Y_{N}=0.4$. As $Y_{\nu}, Y_{N}$ is also determined at the low scale, and its values larger than 0.4 may lead to Landau pole before the GUT scale [33].

In adjusting the ranges of the free parameters, we restrict the scalar and gaugino SSB mass terms not to exceed $5 \mathrm{TeV}$ in order to remain in the regions which yield acceptable fine-tuning at the low scale. Note that we allow different input values for $m_{H_{u}}$ and $m_{H_{d}}$ in our scan, since, as mentioned in Sec. I, they do not reside in the same representation, but they are rather superpositions of the Higgs fields from different representations. The range of the trilinear scalar coupling is set to avoid charge and/or color breaking minima, which requires $\left|A_{0}\right| \lesssim 3 m_{0}$. Among these parameters, we bound $\tan \beta$ at 35 from below. Even though the general MSSM framework can be consistent with the current experimental results including the Higgs boson mass, Yukawa unification requires rather large $\tan \beta$ to satisfy the correct masses for quarks and charged leptons [34]. Even in the case of QYU, the unification scheme needs $\tan \beta \gtrsim 40[12]$.
The REWSB condition puts crucial theoretical constraint [35] on the parameter space given in Eq. (9). According to this constraint, the SSB mass-squared terms for the Higgs doublets are required to be negative at the low scale, though they are positive-defined at $M_{\mathrm{GUT}}$. In this context, the relevant parameters in the RGE evolutions of these mass parameters should be tuned in a way that, $m_{H_{u}}^{2}$ and/or $m_{H_{d}}^{2}$ have to be turn negative. Another constraint is dark matter observations and it restricts the parameter space which requires the lightest supersymmetric particle (LSP) stable and no electric and color charge, which excludes the regions leading to stau or stop LSP solutions [36]. On the other hand, even if a solution does not satisfy the dark matter observations, it can still survive in conjunction with other form(s) of the dark matter formation [37]. Based on this discussion, we accept only the solutions which yield neutralino LSP at the low scale, but we do not apply any constraint from the dark matter experiments.

In scanning the parameter space, we use our interface which employs Metropolis-Hasting algorithm described in [38]. All collected data points satisfy the requirement of REWSB and neutralino LSP. After collecting data, we subsequently impose the mass bounds on all the sparticles [39] and the constraints from rare decay processes $B_{s} \rightarrow$ $\mu^{+} \mu^{-}$[40] and $b \rightarrow s \gamma$ [41]. In addition those bounds we have imposed Higgs boson [42] and gluino masses [43]. The experimental constraints mentioned above can be summarized below:

$$
\begin{aligned}
m_{\tilde{\chi}_{1}^{ \pm}} & \geq 103.5 \mathrm{GeV}, \\
m_{\tilde{\tau}_{1}} & \geq 105 \mathrm{GeV} \\
123 & \leq m_{h} \leq 127 \mathrm{GeV}, \\
m_{\tilde{\tau}} & \geq 105 \mathrm{GeV}, \\
m_{\tilde{g}} & \geq 1800 \mathrm{GeV}, \\
0.8 \times 10^{-9} & \leq \mathrm{BR}\left(B_{s} \rightarrow \mu^{+} \mu^{-}\right) \leq 6.2 \times 10^{-9}(2 \sigma), \\
2.99 \times 10^{-4} & \leq \mathrm{BR}(b \rightarrow s \gamma) \leq 3.87 \times 10^{-4}(2 \sigma)
\end{aligned}
$$

Note that we have employed the LEP bounds on the supersymmetric particles, since these constraints are model independent. However, we leave the details for the current bounds to discuss in Sec. VII. Finally, we identify the regions compatible with QYU by restricting the deviations in the Yukawa couplings within to $20 \%$ by applying $C_{t \nu} \leq$ 0.2 and $C_{b \tau} \leq 0.2$, which refers to the QYU condition.

Besides the constraints and conditions mentioned above, another important constraint on the fundamental parameter space is the cosmological abundance of stable charged particles [44]. It excludes the solutions which yield a charged SUSY particle to be the lightest supersymmetric particle (LSP). In our work, we accept only the solutions for which one of the neutralinos is being the LSP. If LSP neutralino is also assumed to saturate the dark matter relic 

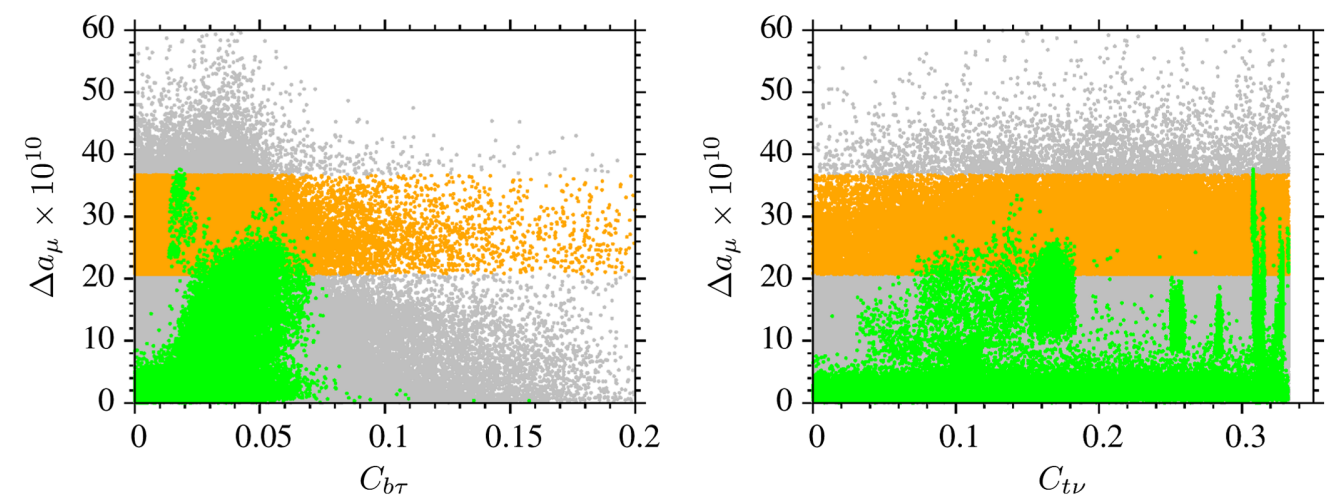

FIG. 2. Plots in the $\Delta a_{\mu}-C_{b \tau}$ and $\Delta a_{\mu}-C_{t \nu}$ planes. All points are consistent with REWSB and neutralino LSP. Green points satisfy the experimental constraints mentioned in Sec. III. Yellow band is an independent subset of gray points, and they indicate the values of $\Delta a_{\mu}$ which would bring the theory and the experiment within $1 \sigma$.

abundance, then one can also apply bounds arising from the dark matter observations. Even though these constraints are also very strong in shaping the fundamental parameter space, solutions excluded by the observations can still provide viable solutions in conjunction with other forms of dark matter [37]. Thus, in our work, we do not require the LSP neutralino to satisfy the WMAP [45] and Planck [46] observations.

\section{FUNDAMENTAL PARAMETER SPACE OF MUON $g-2$ AND SPARTICLE SPECTRUM}

In this section, we discuss muon $g-2$ results and highlight the solutions compatible with the QYU condition. We start first with Fig. 2 displaying the deviations of the Yukawa couplings from the unification with plots in the $\Delta a_{\mu}-C_{b \tau}$ and $\Delta a_{\mu}-C_{t \nu}$ planes. All points are consistent with REWSB and neutralino LSP. Green points satisfy the experimental constraints mention in Sec. III. The yellow band is an independent subset of gray points, and they indicate the values of $\Delta a_{\mu}$ which would bring the theory and the experiment within $1 \sigma$. As seen from the $\Delta a_{\mu}-C_{b \tau}$, $C_{b \tau}$ measuring the deviation in $y_{b}$ and $y_{\tau}$ can barely reach to $20 \%$, and the experimental constraints restrict it to $C_{b \tau} \lesssim$ 0.1 (green). In addition, the region compatible with resolution to muon $g-2$ discrepancy (yellow band) bounds it further to $C_{b \tau} \lesssim 0.07$. On the other hand, $C_{t \nu}$ can be as large as about 0.3 as shown in the $\Delta a_{\mu}-C_{t \nu}$. This is not surprising, since $y_{t}$, and consequently $y_{\nu}$, can receive large corrections from the extra Higgs fields and also the higher dimensional operators. However, it is still possible to restrict it within to $20 \%$. Besides, imposing the QYU condition will exclude the solutions with $C_{t \nu}>0.2$.

Figure 3 represents the correlations between muon $g-2$ and the relevant fundamental parameters with plots in the $\Delta a_{\mu}-m_{0}, \Delta a_{\mu}-M_{2}, \Delta a_{\mu}-M_{1}$ and $\Delta a_{\mu}-\tan \beta$ planes. The color coding is the same as Fig. 2. In addition, blue points form a subset of green and they represent the solutions compatible with the QYU condition. The
$\Delta a_{\mu}-m_{0}$ shows that $m_{0}$ cannot be greater than about $1.2 \mathrm{TeV}$ in order for the resolution to muon $g-2$ discrepancy. Since $m_{0}$ controls the scalar masses, it is understandable with the need of light scalars, which run in the loops contributing to muon $g-2$, at the low scale. However, the regions with $m_{0} \lesssim 600 \mathrm{GeV}$ cannot provide a resolution to muon $g-2$ consistently accommodated with the current experimental constraints. This result arises from the effects of the right-handed neutrino sector discussed along with the RGEs in the previous section. The gray region coinciding with the yellow band yield inconsistently light charged sleptons $\left(m_{\tilde{l}}<100 \mathrm{GeV}\right)$ especially for those from the third family. In addition, the Higgs boson mass is problematic in these regions, since most of the solutions predict $m_{h}<125 \mathrm{GeV}$. Similarly, muon $g-2$ condition requires light weakinos (bino and wino), and $\left|M_{2}\right| \lesssim$ $500 \mathrm{GeV}$ as seen from the $\Delta a_{\mu}-M_{2}$ plane. This parameter controls the wino mass at the low scale as $m_{\tilde{W}} \approx\left|M_{2}\right|$; hence, muon $g-2$ condition necessitates light charginos at the low scale. Similarly, $M_{1}$, which controls the Bino mass as $m_{\tilde{B}} \approx 0.5\left|M_{1}\right|$ [47], needs to be light $(\lesssim 500 \mathrm{GeV})$. Since we set in $\mu<0$, one can expect to have large SUSY contributions to muon $g-2$ when $M_{1}$ is negative in contrast to the results shown in the $\Delta a_{\mu}-M_{1}$ plane, where the SUSY contributions seem suppressed when $M_{1}<0$.

We consider the low-scale mass spectrum for the supersymmetric particles in Fig. 4 with plots in the $\Delta a_{\mu}-m_{\tilde{\chi}_{1}^{0}}$, $\Delta a_{\mu}-m_{\tilde{\chi}_{1}^{ \pm}}, \Delta a_{\mu}-m_{\tilde{\mu}_{1}}$ and $\Delta a_{\mu}-m_{\tilde{\nu}_{1}}$ planes. The color coding is the same as Fig. 3. The neutralino LSP mass cannot exceed about $200 \mathrm{GeV}$ in order for maintaining the resolution to muon $g-2$, while the lightest chargino can be as heavy as about $400 \mathrm{GeV}$ as seen from the $\Delta a_{\mu}-m_{\tilde{\chi}_{1}^{ \pm}}$. In contrast to the usual MSSM implications, the IS mechanism yields rather heavy smuons $\left(m_{\tilde{\mu}} \gtrsim 800 \mathrm{GeV}\right)$, which can significantly suppress the SUSY contributions from the smuon-neutralino loop processes. As discussed above, the smuon masses are mostly bounded from below by the stau mass, which can turn to be tachyonic due to its large 

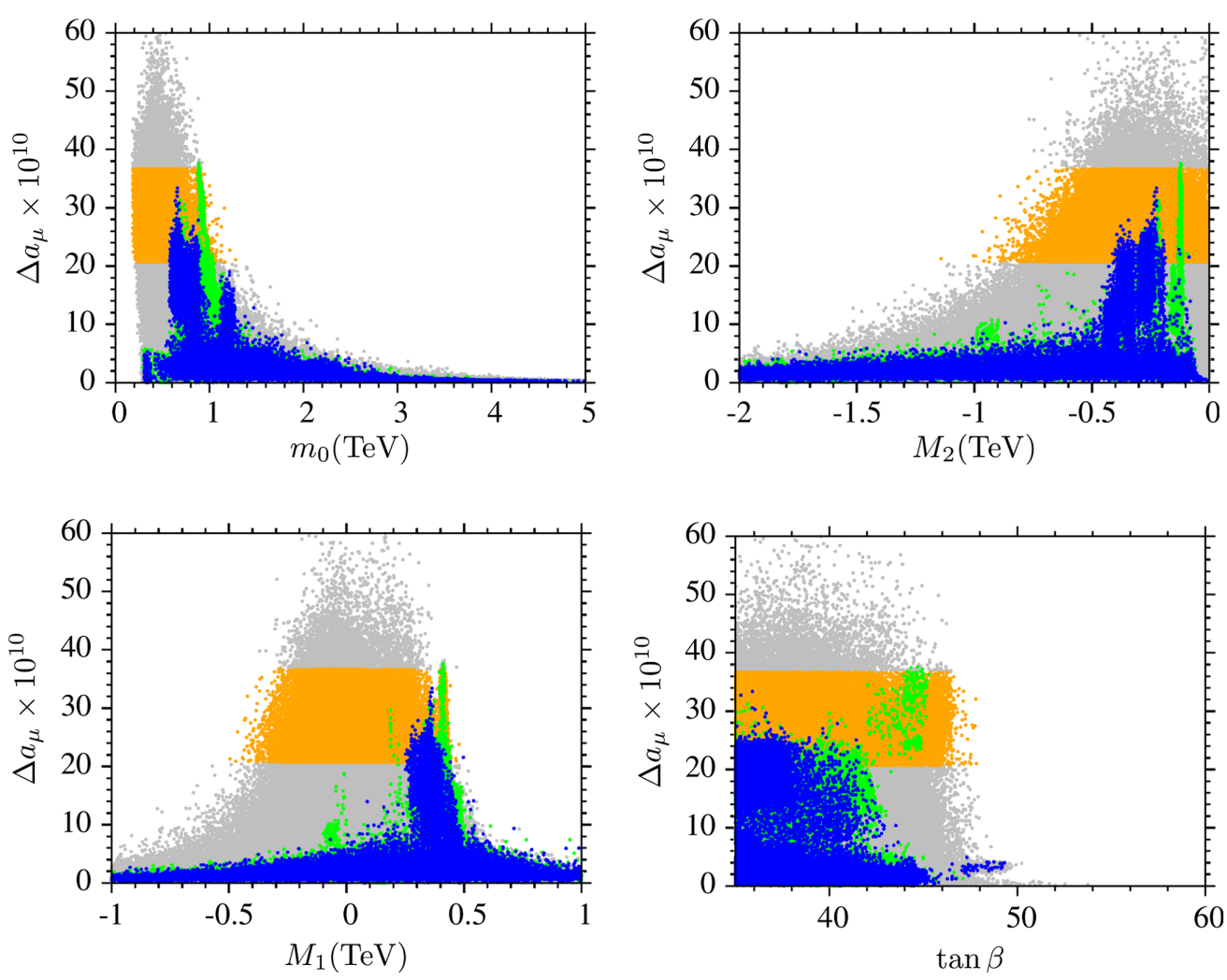

FIG. 3. Plots in the $\Delta a_{\mu}-m_{0}, \Delta a_{\mu}-M_{2}, \Delta a_{\mu}-M_{1}$ and $\Delta a_{\mu}-\tan \beta$ planes. The color coding is the same as Fig. 2. In addition, blue points form a subset of green and they represent the solutions compatible with the QYU condition.
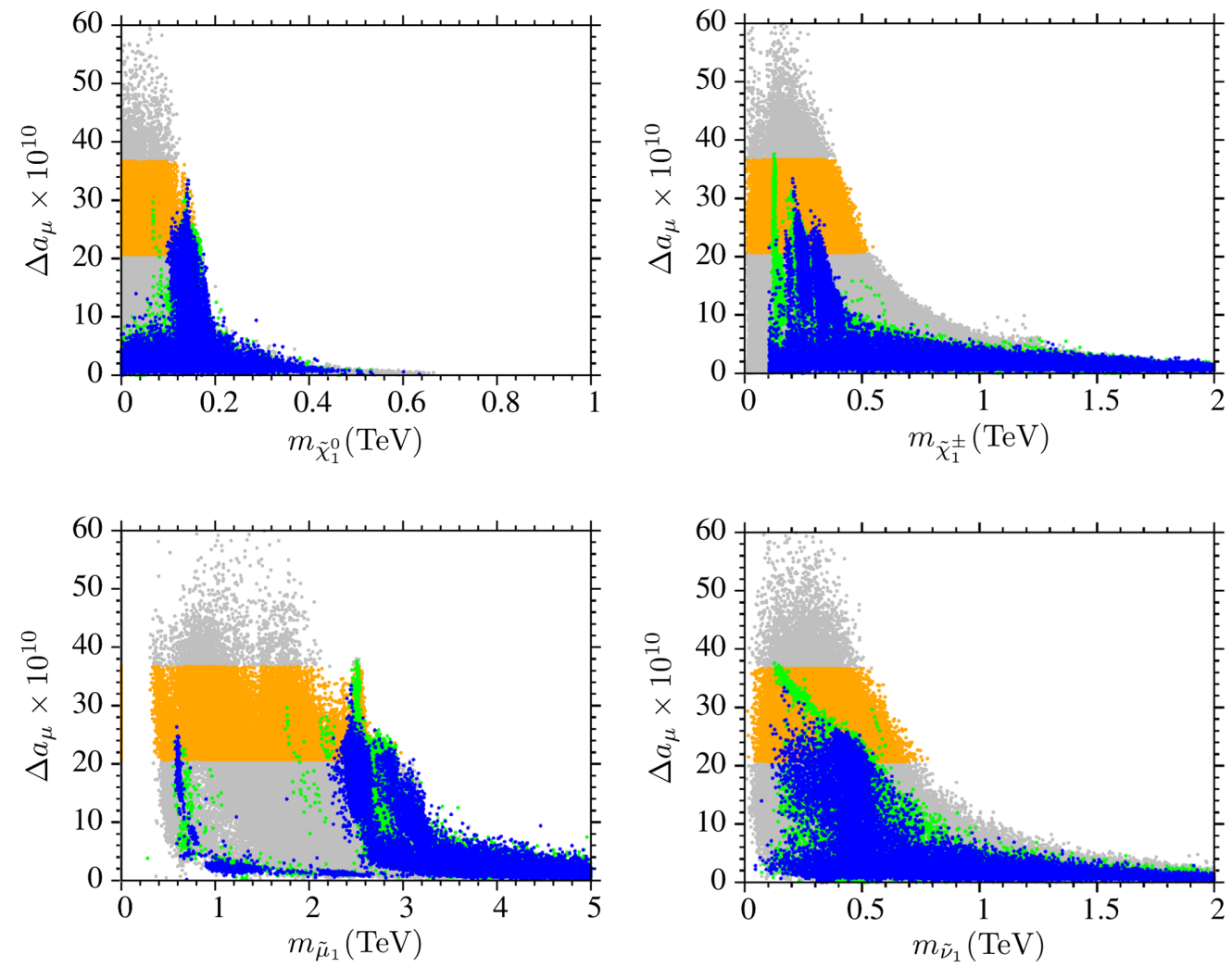

FIG. 4. Plots in the $\Delta a_{\mu}-m_{\tilde{\chi}_{1}^{0}}, \Delta a_{\mu}-m_{\tilde{\chi}_{1}^{ \pm}}, \Delta a_{\mu}-m_{\tilde{\mu}_{1}}$ and $\Delta a_{\mu}-m_{\tilde{\nu}_{1}}$ planes. The color coding is the same as Fig. 3. 
trilinear SSB term $\left(T_{\tau} \equiv A_{\tau} y_{\tau}\right)$, when the other charged sleptons are light. However, there is a narrow blue stripe in this region, which provide large enough SUSY contributions to muon $g-2$ and satisfy the mass bound on the charged sleptons. In this region, smuon-neutralino loop is able to provide minor but comparable contributions in resolution to the muon $g-2$ problem. While the stau mass bound has a strong impact on the smuon masses, the sneutrinos, on the other hand, can be as light as about $100 \mathrm{GeV}$, which yield significant SUSY contributions to muon $g-2$ along with light charginos. Consequently, the main contributions to muon $g-2$ are provided from the sneutrino-chargino loop processes, while those from smuon and neutralino are either suppressed or minor due to the heavy smuon masses. This also explains why there is no significant muon $g-2$ contributions when $M_{1}$ is negative. In this region, $M_{2}$ needs to be larger than $M_{3}$, which yields relatively heavy charginos at the low scale, so the SUSY contributions from chargino and sneutrino are also suppressed. In addition, the reason why the neutralino mass is bounded from above as $m_{\tilde{\chi}_{1}^{0}} \lesssim 200 \mathrm{GeV}$ is only the condition which requires neutralino to be LSP for all the solutions.

\section{SPARTICLE SPECTRUM}

In the previous section, we highlighted the results in respect of muon $g-2$ and masses of the relevant sparticles. In this section, we present the sparticle mass spectrum in more details. Figure 5 displays our results with the plots in the $m_{\tilde{\mu}_{1}}-m_{\tilde{\tau}_{1}}, m_{\tilde{\mu}_{1}}-m_{\tilde{\nu}_{1}}, m_{\tilde{\chi}_{1}^{ \pm}}-m_{\tilde{\chi}_{1}^{0}}$, and $M_{2} / \mu-M_{1} / \mu$. All points are consistent with REWSB and LSP neutralino. Green points are allowed by the experimental constraints. Blue points form a subset of green, and they satisfy the QYU condition. Orange points are a subset of blue and they represent the solutions yielding muon $g-2$ within $1 \sigma$. The diagonal line in the $M_{2} / \mu-M_{1} / \mu$ plane indicates the regions where $M_{2} \approx M_{1}$. As mentioned before, avoiding tachyonic stau mass solutions bounds the smuon mass from below, and the $m_{\tilde{\mu}_{1}}-m_{\tilde{\tau}_{1}}$ plane, most of the solutions lead to smuons heavier than about $1 \mathrm{TeV}$ in orange region. Such heavy smuon solutions suppress the SUSY contributions from smuon-neutralino loops; thus, the significant muon $g-2$ contributions in this region mostly rely on sneutrinochargino loops as diagrammatically shown in Fig. 1. On the other hand, it is still possible to realize solutions compatible with the muon $g-2$ results within $1 \sigma$ when $m_{\tilde{\mu}_{1}} \gtrsim$ $600 \mathrm{GeV}$ without leading to the tachyonic stau situation.
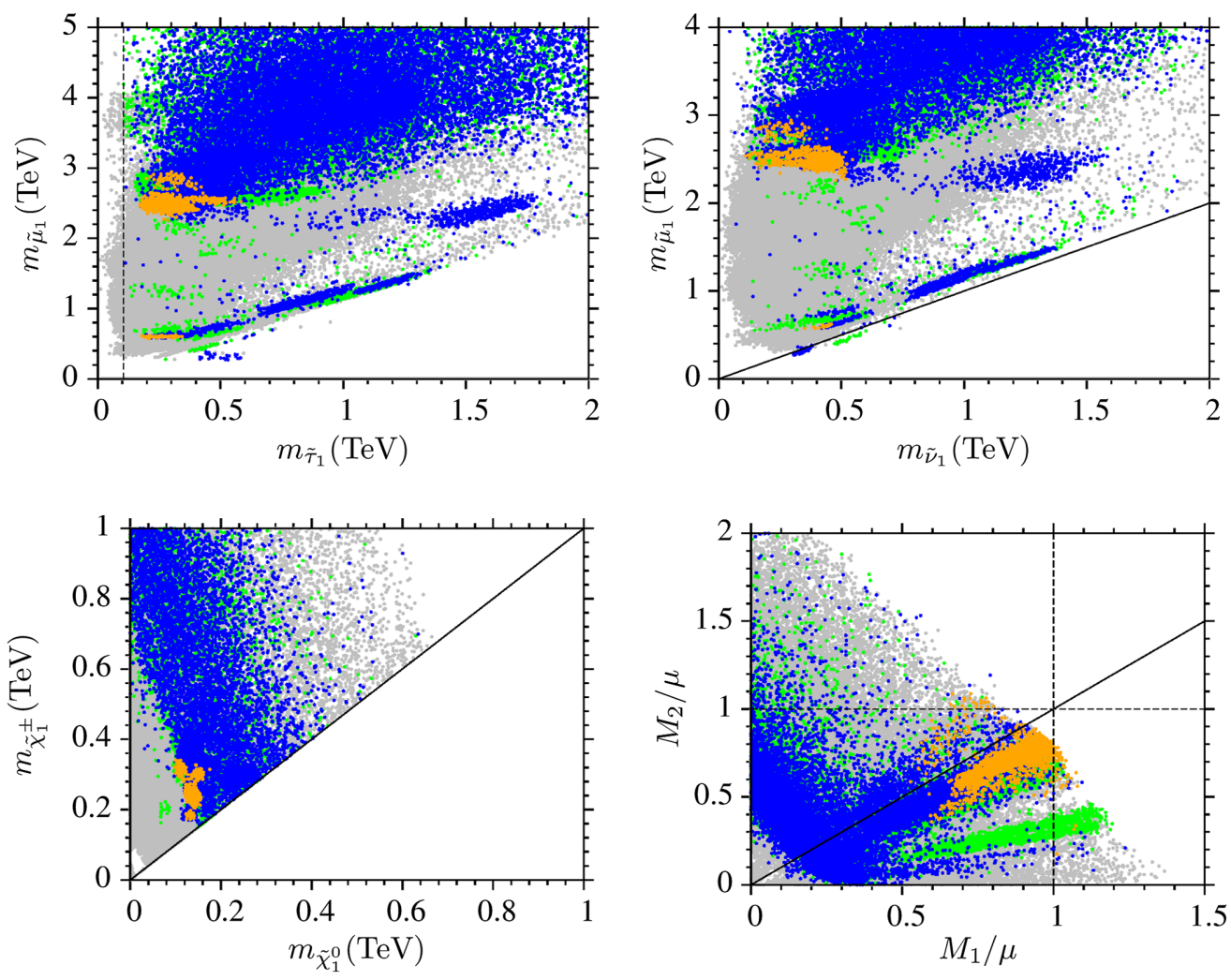

FIG. 5. Plots in the $m_{\tilde{\mu}_{1}}-m_{\tilde{\tau}_{1}}, m_{\tilde{\mu}_{1}}-m_{\tilde{\nu}_{1}}, m_{\tilde{\chi}_{1}^{ \pm}}-m_{\tilde{\chi}_{1}^{0}}$, and $M_{2} / \mu-M_{1} / \mu$. All points are consistent with REWSB and LSP neutralino. Green points are allowed by the experimental constraints. Blue points form a subset of green, and they satisfy the QYU condition. Orange points are a subset of blue and they represent the solutions yielding muon $g-2$ within $1 \sigma$. The diagonal line in the $M_{2} / \mu-M_{1} / \mu$ plane indicates the regions where $M_{2} \approx M_{1}$. 

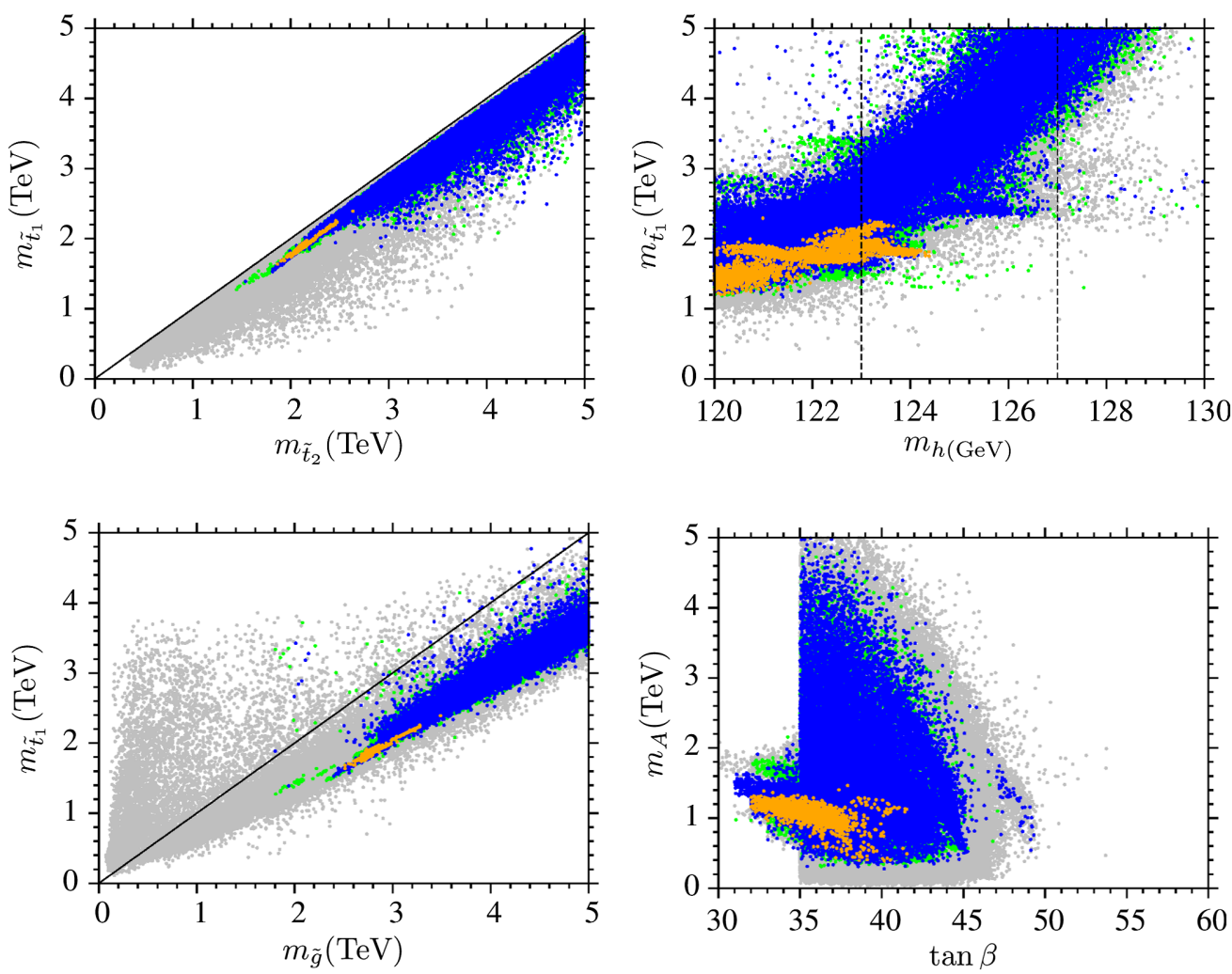

FIG. 6. Plots in the $m_{\tilde{t}_{1}}-m_{\tilde{t}_{2}}, m_{\tilde{t}_{1}}-m_{h}, m_{\tilde{t}_{1}}-m_{\tilde{g}}$, and $m_{A}-\tan \beta$. The color coding is the same as Fig. 5. The diagonal line in the $m_{\tilde{t}_{1}}-m_{\tilde{t}_{2}}$ indicates the regions where $m_{\tilde{t}_{1}}=m_{\tilde{t}_{2}}$.

These solutions can be realized when the terms with $T_{\nu}$ in RGEs given in Eq. (8) are small. Since $T_{\nu}$ is determined with $A_{0}$ at the GUT scale as all the other trilinear scalar interaction couplings, solutions in the region with relatively lighter smuons also have small $A$ terms, which is favored in acceptable fine-tuning considerations [26]. We consider the smuon mass also in compared to the sneutrino mass in the $m_{\tilde{\mu}_{1}}-m_{\tilde{\nu}_{1}}$ plane. The sneutrino mass is bounded as $m_{\tilde{\nu}_{1}} \lesssim 600 \mathrm{GeV}$, even though the smuons are also light. Such results show that the contributions from the sneutrinochargino loops are necessary in realizing the muon $g-2$ solutions within $1 \sigma$.

The other supersymmetric particles playing a key role in the muon $g-2$ are the chargino and neutralino whose masses are represented in the $m_{\tilde{\chi}_{1}^{ \pm}}-m_{\tilde{\chi}_{1}^{0}}$ plane. The muon $g-2$ results restrict these particles as $m_{\tilde{\chi}_{1}^{ \pm}} \lesssim 400 \mathrm{GeV}$ and $m_{\tilde{\chi}_{1}^{0}} \lesssim 200 \mathrm{GeV}$ in order to overcome mass suppression in SUSY contributions to muon $g-2$. The last panel represents the LSP neutralino composition in terms of the ratio of the gaugino masses to the $\mu$ term. Since most of the solutions represented with orange are accumulated below the diagonal line and $M_{1} / \mu<1$, the LSP neutralino is formed mostly by Bino in this region, while wino significantly mixes with bino in LSP neutralino formation near the diagonal line. There are also a considerable number of solutions (orange) above the diagonal line, which yield mostly winolike LSP neutralino. Since all orange solutions are found in the regions either with $M_{1} / \mu<1$ or $M_{2} / \mu<1$, Higgsinos are not much effective in the LSP neutralino composition.

Even though they do not take a direct part in calculation of the SUSY contributions to muon $g-2$, we present other sparticle masses for completeness in Fig. 6 with plots in the $m_{\tilde{t}_{1}}-m_{\tilde{t}_{2}}, m_{\tilde{t}_{1}}-m_{h}, m_{\tilde{t}_{1}}-m_{\tilde{g}}$, and $m_{A}-\tan \beta$. The color coding is the same as Fig. 5. The diagonal line in the $m_{\tilde{t}_{1}}-$ $m_{\tilde{t}_{2}}$ indicates the regions where $m_{\tilde{t}_{1}}=m_{\tilde{t}_{2}}$. The $m_{\tilde{t}_{1}}-m_{\tilde{t}_{2}}$ plane shows that the stop cannot be lighter than about $1 \mathrm{TeV}$. In addition the lightest and heaviest stop masses in the orange region are comparable to each other $\left(m_{\tilde{t}_{2}} \gtrsim m_{\tilde{t}_{1}}\right)$. As mentioned in discussion about smuon and stau masses, small $A$ terms yield small mixture between the right-handed and left-handed stop states, and their masses can be realized closed to each other. Even though the presence of righthanded neutrinos may relax the heavy stop condition to realize a $125 \mathrm{GeV}$ Higgs boson, the stop mass scale is still strongly affected by the Higgs boson mass condition as seen from the $m_{\tilde{t}_{1}}-m_{h}$. This is also because of the small $A$ terms. Since the Higgs boson mass does not receive large enough contributions from the stop mixing, solutions consistent with the Higgs boson constraint still rely on the stop mass, and they require $m_{\tilde{t}_{1}} \gtrsim 1 \mathrm{TeV}$. In addition to stop, the gluino mass scale is also quite interesting since it 

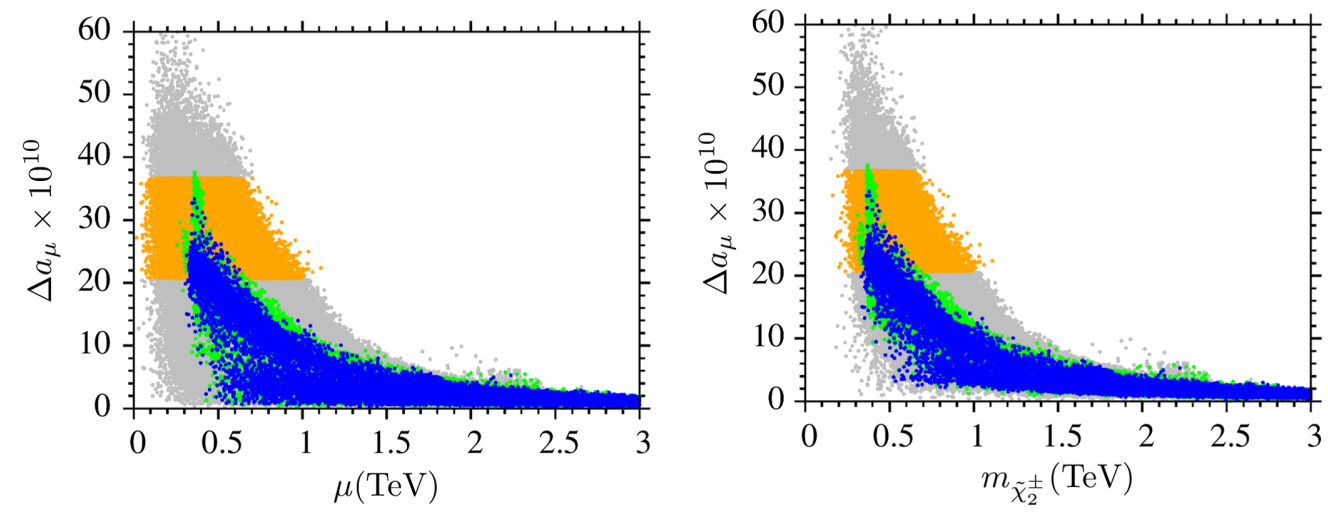

FIG. 7. Plots in the $\Delta a_{\mu}-\mu$ and $\Delta a_{\mu}-m_{\tilde{\chi}_{2}^{ \pm}}$planes. The color coding is the same as Fig. 3.

lies from about 2 to $4 \mathrm{TeV}$. In this context, even though the current mass bound on gluino is slightly larger $\left(m_{\tilde{g}} \geq 1.9 \mathrm{TeV}\right.$ [48]) than what we applied, the muon $g-2$ condition itself exclude gluino solutions excluded by the current LHC results. In addition, the solutions with $m_{\tilde{g}} \gtrsim$ $2.5 \mathrm{TeV}$ provide testable results in the current or near future experiments, since the next generation of colliders are planned to probe the gluino mass up to about $3 \mathrm{TeV}$ [49]. Finally we present the $C P$-odd Higgs boson mass in correlation with $\tan \beta$ in the $m_{A}-\tan \beta$ plane. Its mass is bounded from above as $m_{A} \lesssim 1.4 \mathrm{TeV}$ by the muon $g-2$ condition for moderate $\tan \beta$, while it decreases as $\tan \beta$ increases. The solutions with $m_{A}<1 \mathrm{TeV}$ in the large $\tan \beta$ region can be constrained further by detailed analyses [50].

Note that the orange points are compatible with all the constraints listed in Section III, and the QYU and $\Delta a_{\mu}$ conditions simultaneously. The only exception is the topright panel of Fig. 6, which represents our results in the $m_{\tilde{t}_{1}}-m_{h}$ plane. Since the Higgs boson mass is directly plotted on the horizontal axis, we have not employed the constraint from the Higgs boson mass; rather we have used two vertical lines which bound the allowed region by the Higgs boson mass. Even though the allowed solutions occupy a small region in this plane, they can spread more in the other plots and the allowed region can seem larger. Employing the Higgs boson mass constraint in the other plots remove the orange points outside the region between the two vertical lines, which are excluded due to yielding inconsistent Higgs boson mass. Hence these solutions are not represented in the other plots.

\section{FINE-TUNING AND MUON $g-2$ IN MSSM WITH IS}

As we discussed before, the dominant contribution to muon $g-2$ comes from the sneutrino-chargino loop processes. In these processes, the chargino can be either wino or Higgsino, each of which corresponds to different nature of the SUSY contributions. If the chargino is winolike, then the contributions are generated through
$S U(2)$ interactions, while the Yukawa interactions take part when the chargino is Higgsino-like. Depending on the ratios of their masses, the chargino could also be a mixture of these two particles. Figure 7 represents the result for the Higgsino and heaviest chargino masses with plots in the $\Delta a_{\mu}-\mu$ and $\Delta a_{\mu}-m_{\tilde{\chi}_{2}^{ \pm}}$planes. The color coding is the same as Fig. 3. According to the $\Delta a_{\mu}-\mu$ plane, the Higgsinos, whose masses are equal to $\mu$, can be as light as about $500 \mathrm{GeV}$, while muon $g-2$ condition bounds its mass from above at about $700 \mathrm{GeV}$. In this sense, the model predicts relatively light Higgsinos at the low scale compatible with the QYU condition. If the low-scale spectrum includes two charginos lighter than about a $\mathrm{TeV}$, then the processes can contribute to muon $g-2$, even when the heaviest chargino runs in the loop. Even though the heaviest chargino contribution can only be minor in comparison to the lightest chargino contribution, its mass cannot be heavier than about $800 \mathrm{GeV}$ for the resolution to muon $g-2$ discrepancy, as seen from the $\Delta a_{\mu}-m_{\tilde{\chi}_{2}^{ \pm}}$.

The light Higgsinos are also interesting from the naturalness point of view. Since the mass bounds on the supersymmetric particles become severe after the latest results from the LHC experiments, the solutions can barely be placed in the natural region characterized with $m_{\tilde{t}_{1}}, m_{\tilde{t}_{2}}, m_{\tilde{b}_{1}} \lesssim 500 \mathrm{GeV}$. Especially the Higgs boson mass constraint requires at least one stop to have mass above $\mathrm{TeV}$ scale. On the other hand, deviation from the natural region can be measured with $\Delta_{\mathrm{EW}}$, the fine-tuning parameter as defined in Ref. [26]. $\Delta_{\mathrm{EW}}$ is a function of $\mu, m_{H_{d}}, m_{H_{u}}$, and $\tan \beta$, in principal; however, the terms proportional to $m_{H_{d}}$ are suppressed by $\tan \beta$, and the correct electroweak symmetry breaking scale requires $\mu \approx m_{H_{u}}$ over most of the fundamental parameter space. In this sense, the Higgsino masses can also indicate the fine-tuning amount required to have consistent electroweak symmetry breaking. Since our model predicts relatively light Higgsinos $(\lesssim 800 \mathrm{GeV})$ compatible with the resolution to muon $g-2$ discrepancy, such solutions also need low fine-tuning. In general fashion, the acceptable fine-tuning is identified 

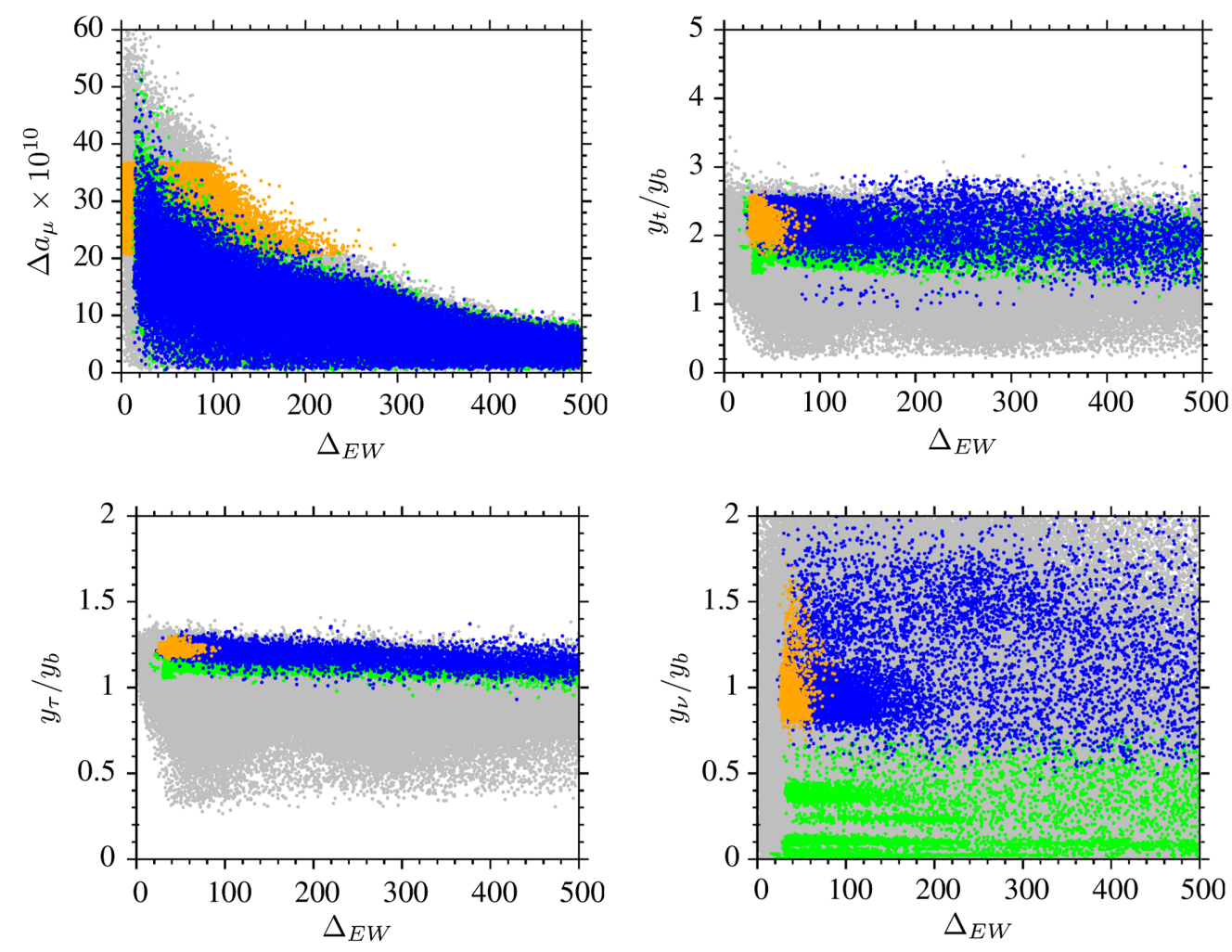

FIG. 8. Plots in the $\Delta a_{\mu}-\Delta_{\mathrm{EW}}, y_{t} / y_{b}-\Delta_{\mathrm{EW}}, y_{\tau} / y_{b}-\Delta_{\mathrm{EW}}$ and $y_{\nu} / y_{b}-\Delta_{\mathrm{EW}}$ planes. The color coding is the same as Fig. 3.

with the condition $\Delta_{\mathrm{EW}} \leq 1000$. Figure 8 investigates our results about the fine-tuning with plots in the $\Delta a_{\mu}-\Delta_{\mathrm{EW}}$, $y_{t} / y_{b}-\Delta_{\mathrm{EW}}, y_{\tau} / y_{b}-\Delta_{\mathrm{EW}}$ and $y_{\nu} / y_{b}-\Delta_{\mathrm{EW}}$ planes. The color coding is the same as Fig. 3. As seen from the $\Delta a_{\mu}-$ $\Delta_{\text {EW }}$ plane, $\Delta_{\mathrm{EW}}$ can be as low as 20 compatible with muon $g-2$ condition. Indeed, muon $g-2$ condition restricts $\Delta_{\text {EW }} \lesssim 100$, and the discrepancy cannot be solved within $1 \sigma$ when $\Delta_{\mathrm{EW}}>100$. In addition to muon $g-2$ resolution, we also discuss the Yukawa couplings, whose deviations are also restricted by the QYU condition in the $y_{t} / y_{b}-\Delta_{\mathrm{EW}}$, $y_{\tau} / y_{b}-\Delta_{\mathrm{EW}}$ and $y_{\nu} / y_{b}-\Delta_{\mathrm{EW}}$ planes. According to the results represented in these planes, $y_{t} / y_{b} \gtrsim 2, y_{\tau} / y_{b} \gtrsim 1.2$, and $y_{\nu} / y_{b} \gtrsim 0.8$ over the region with the acceptable finetuning.

\section{THE LIGHT CHARGINOA IN LHC}

As discussed in the previous sections, the resolution to the muon $g-2$ can be accommodated with the QYU in the presence of the right-handed neutrinos and inverse seesaw mechanism. However, such a resolution requires rather light chargino, neutralino and stau. Even though we have employed the LEP bounds on these supersymmetric particle masses, the new analyses $[51,52]$ have shown that such solutions might be excluded. The severest bound on the chargino mass has been obtained when the charged sleptons are light enough that the chargino and/or the second lightest neutralino are kinematically allowed to decay into them. In such cases, the solutions with $m_{\tilde{\chi}_{1}^{ \pm}} \lesssim 1.1 \mathrm{TeV}$ are excluded for $m_{\tilde{\chi}_{1}^{0}} \lesssim 700 \mathrm{GeV}$ [51]. Even though the mass bound on the chargino is loosen as $m_{\tilde{\chi}_{1}^{ \pm}} \gtrsim 500 \mathrm{GeV}$ [52] when the sleptons are heavy, such exclusion bounds are based on the decay modes involving with the $W^{ \pm}$or $H$ bosons, where $H$ is the heavier $C P$-even Higgs boson of MSSM.

Even though the low-scale analyses allow one to fix the configuration out of more than a hundred free parameters, in which the relevant decay modes of the particles can be set to their maximum values, such configurations may not be possible when the models are constrained from the GUT scale with a few free parameters. Hence, before proceeding to apply these constraints, one should check which case can be applicable to data in order to analyze the low-scale implications. We present the masses of the relevant supersymmetric particles in Fig. 9 with plots in the $m_{\tilde{\tau}_{L}}-m_{\tilde{\chi}_{1}^{ \pm}}$, $m_{\tilde{\tau}_{R}}-m_{\tilde{\chi}_{1}^{ \pm}}, m_{\tilde{\nu}}-m_{\tilde{\chi}_{1}^{ \pm}}$and $m_{\tilde{\chi}_{1}^{ \pm}}-m_{\tilde{\chi}_{1}^{0}}$ planes. All points are consistent with REWSB and LSP neutralino. Green points are allowed by the experimental constraints. Blue points form a subset of green, and they satisfy the QYU condition. Orange points are a subset of blue and they represent the solutions yielding muon $g-2$ within $1 \sigma$. The diagonal lines indicate the mass degeneracy between the plotted particles. In addition, the upper diagonal line in the $m_{\tilde{\chi}_{1}^{ \pm}}-$ $m_{\tilde{\chi}_{1}^{0}}$ plane represents the region where $m_{\tilde{\chi}_{1}^{ \pm}}=m_{\tilde{\chi}_{1}^{0}}+m_{W^{ \pm}}$, where $m_{W^{ \pm}}$denotes the $W^{ \pm}$mass. Note that the LEP bounds are still employed on the masses. 

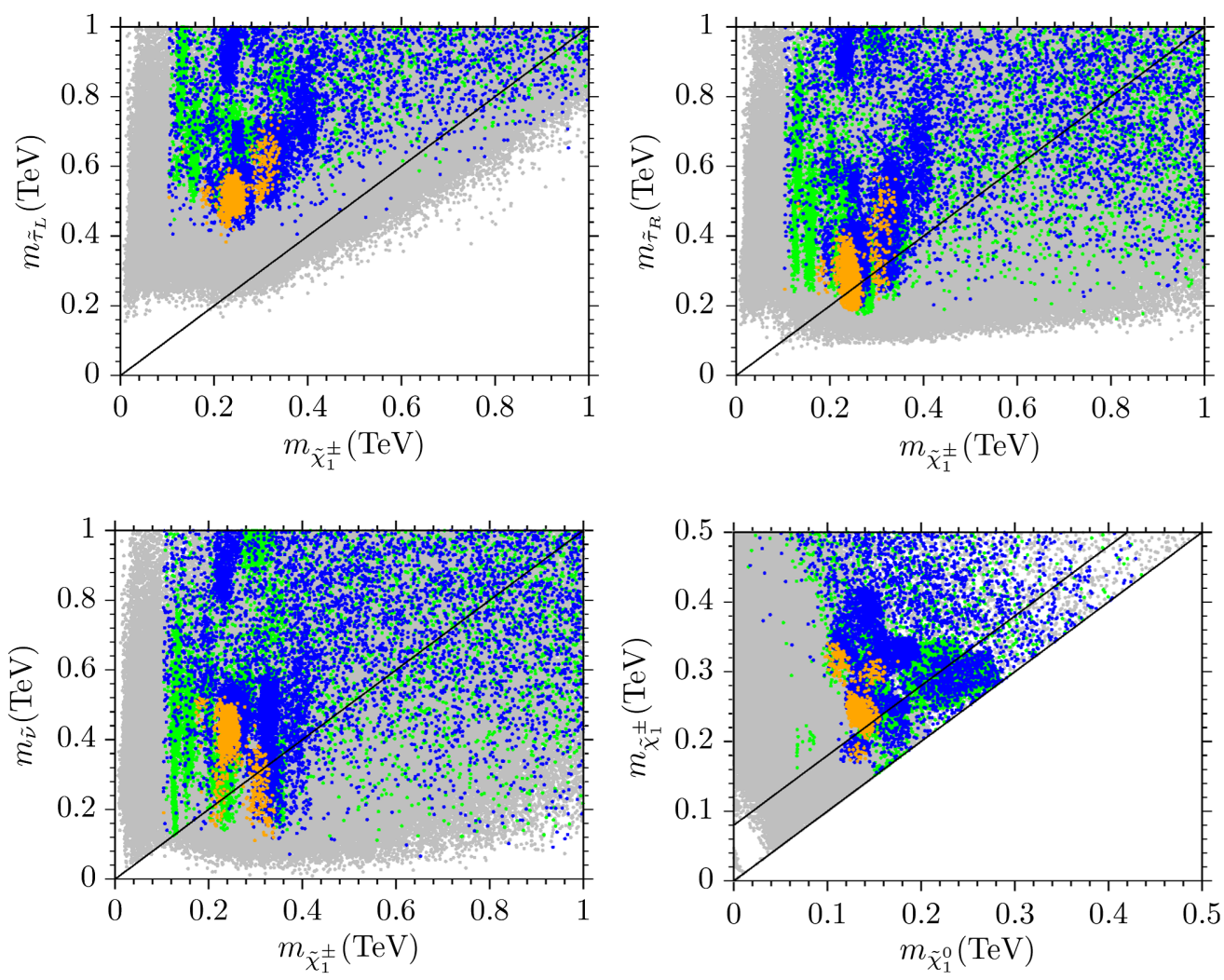

FIG. 9. Plots in the $m_{\tilde{\tau}_{L}}-m_{\tilde{\chi}_{1}^{ \pm}}, m_{\tilde{\tau}_{R}}-m_{\tilde{\chi}_{1}^{ \pm}}, m_{\tilde{\nu}}-m_{\tilde{\chi}_{1}^{ \pm}}$and $m_{\tilde{\chi}_{1}^{ \pm}}-m_{\tilde{\chi}_{1}^{0}}$ planes. All points are consistent with REWSB and LSP neutralino. Green points are allowed by the experimental constraints. Blue points form a subset of green, and they satisfy the QYU condition. Orange points are a subset of blue and they represent the solutions yielding muon $g-2$ within $1 \sigma$. The diagonal lines indicate the mass degeneracy between the plotted particles. In addition, the upper diagonal line in the $m_{\tilde{\chi}_{1}^{ \pm}}-m_{\tilde{\chi}_{1}^{0}}$ plane represents the region where $m_{\tilde{\chi}_{1}^{ \pm}}=m_{\tilde{\chi}_{1}^{0}}+m_{W^{ \pm}}$, where $m_{W^{ \pm}}$denotes the $W^{ \pm}$mass.

The $m_{\tilde{\tau}_{L}}-m_{\tilde{\chi}_{1}^{ \pm}}$plane shows that there is no solution in which the left-handed slepton is lighter than the chargino, while it is possible to realize lighter right-handed slepton in a small portion of the parameter space (orange points below the diagonal line) as is seen in the $m_{\tilde{\tau}_{R}}-m_{\tilde{\chi}_{1}^{ \pm}}$plane. Following the discussion in Ref. [51], when only the right-handed slepton can be lighter than the chargino, then the chargino can only decay into a $\tau$ lepton and a sneutrino. On the other hand, the second lightest neutralino is still allowed to decay into charged sleptons. In this region, also sneutrinos are light enough that the chargino can decay into them. As seen from the results many solutions yield charged sleptons heavier. In this region, the chargino can decay into the LSP neutralino with a $W^{ \pm}$boson, while the second chargino can decay into the LSP neutralino along with a $Z$ or $H$ boson, where $H$ denotes the heavier $C P$-even Higgs boson of MSSM. However, there are also some solutions, seen between the two diagonal lines in the $m_{\tilde{\chi}_{1}^{ \pm}}-$ $m_{\tilde{\chi}_{1}^{0}}$ plane, do not allow $\tilde{\chi}_{1}^{ \pm} \rightarrow W^{ \pm} \tilde{\chi}_{1}^{0}$. In this region, the chargino can decay into the LSP neutralino along with quarks of the first two families, and the mass bounds mentioned above are not applicable to such solutions, since the uncertainty in the QCD sector is relatively large.
After all, the mass bound $m_{\tilde{\chi}_{1}^{ \pm}} \gtrsim 1.1 \mathrm{TeV}$ can be applicable in a small region which is shown below the diagonal line in the $m_{\tilde{\tau}_{R}}-m_{\tilde{\chi}_{1}^{ \pm}}$plane of Fig. 9. A further assumption behind the detector analyses is to fix the second lightest neutralino decay mode as $\operatorname{BR}\left(\tilde{\chi}_{2}^{0} \rightarrow \tilde{\tau} \tau\right)=0.5$. When we check if this assumption is satisfied in the relevant region, the results are obtained for the relevant decay modes of chargino and the second lightest neutralino as shown in Fig. 10. The color coding is the same as Fig. 9. In addition, the brown points in the $m_{\tilde{\tau}_{R}}-m_{\tilde{\chi}_{1}^{ \pm}}$form a subset of orange, and they satisfy the condition $\operatorname{BR}\left(\tilde{\chi}_{2}^{0} \rightarrow \tilde{\tau} \tau\right) \geq 0.3$. As is seen from the $\operatorname{BR}\left(\tilde{\chi}_{1}^{ \pm} \rightarrow \tilde{\nu} \tau\right)-\operatorname{BR}\left(\tilde{\chi}_{2}^{0} \rightarrow \tilde{\tau} \tau\right)$ plane almost all orange points yield $\operatorname{BR}\left(\tilde{\chi}_{1}^{ \pm} \rightarrow \tilde{\nu} \tau\right) \approx 0.5$. On the other hand, only a small portion of such solutions can yield large $\operatorname{BR}\left(\tilde{\chi}_{2}^{0} \rightarrow \tilde{\tau} \tau\right)$. If a solution yields $\operatorname{BR}\left(\tilde{\chi}_{2}^{0} \rightarrow \tilde{\tau} \tau\right) \lesssim 30 \%$, it reduces the signal strength at least $40 \%$. The brown points in the $m_{\tilde{\tau}_{R}}-m_{\tilde{\chi}_{1}^{ \pm}}$plane represents the solutions with $\operatorname{BR}\left(\tilde{\chi}_{2}^{0} \rightarrow \tilde{\tau} \tau\right) \lesssim 30 \%$, and as is seen, there are still some solutions with $m_{\tilde{\tau}_{R}} \lesssim m_{\tilde{\chi}_{1}^{ \pm}}$, and they can survive under the current constraints on the chargino.

Based on the discussion above, one can conclude that the solutions yielding $\operatorname{BR}\left(\tilde{\chi}_{2}^{0} \rightarrow \tilde{\tau} \tau\right) \geq 0.3$ can be considered 

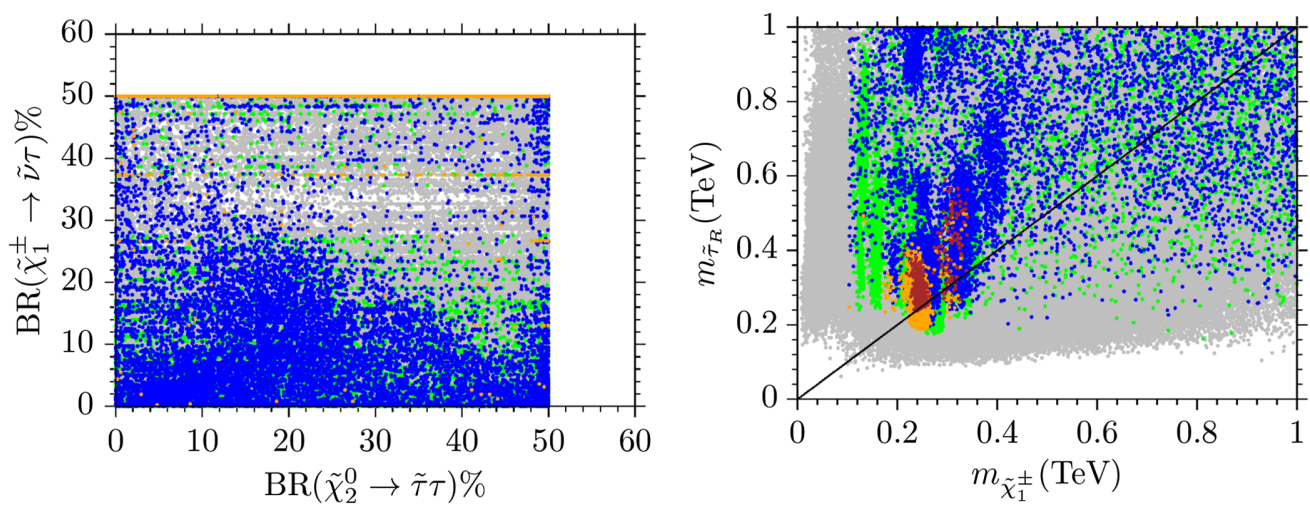

FIG. 10. Results in the $\operatorname{BR}\left(\tilde{\chi}_{1}^{ \pm} \rightarrow \tilde{\nu} \tau\right)-\operatorname{BR}\left(\tilde{\chi}_{2}^{0} \rightarrow \tilde{\tau} \tau\right)$ and $m_{\tilde{\tau}_{R}}-m_{\tilde{\chi}_{1}^{ \pm}}$planes. The color coding is the same as Fig. 9. In addition, the brown points in the $m_{\tilde{\tau}_{R}}-m_{\tilde{\chi}_{1}^{ \pm}}$form a subset of orange, and they satisfy the condition $\operatorname{BR}\left(\tilde{\chi}_{2}^{0} \rightarrow \tilde{\tau} \tau\right) \geq 0.3$.

potentially excluded. However, such a bound is only conventional and based on the assumption that the chargino-neutralino production rate is equal to that employed in the detector analyses. In this context, even though we exclude such solutions, they still provide testable solutions and the analyses should be completed by performing the chargino-neutralino production rate calculation. A recent analyze represented in, for instance, Ref. [53] can exclude the chargino solutions only up to about $700 \mathrm{GeV}$, although the analyses were performed without constraining MSSM from the GUT scale.

Figure 9 shows that the sleptons are heavy over most of the fundamental parameter space, and hence, they cannot take part in decay modes of the chargino and neutralino. In this case, the exclusion bound on the chargino and neutrino masses can be obtained from the decay modes in which the chargino decays into a $W^{ \pm}$boson along with a lightest neutralino, while the second lightest neutralino can decay into either of a $Z$ and $H$ bosons. However, the mass bounds from these decay modes are not as severe as the others considered above, and they can exclude the chargino mass below about $600 \mathrm{GeV}$ [52]. It is interesting for these channels that the exclusion on the chargino mass is not sensitive when $m_{\tilde{\chi}_{1}^{ \pm}} \lesssim 180 \mathrm{GeV}$ [52]. This is because the relevant background involving with $W^{ \pm}$boson is large and it requires large cuts on the missing energy to suppress the background processes. If we apply the exclusion curve given in [52] on the chargino and lightest neutralino mass plane, the results are obtained as plotted in Fig. 11. The color coding is the same as Fig. 9. The diagonal line indicates the mass degeneracy between the lightest chargino and neutralino. The solid black curve represents the exclusion curve obtained in [52]. As one can see, even though we assume the possible largest chargino-neutralino production rates and branching ratios for the decays, only a small portion of orange solutions can be excluded. As stated before, these points can still be available and testable, when the chargino-neutralino production rate is employed in the analyses.
Even though the lightest chargino can still be allowed or testable under the current analyses, our analyses show that the second chargino is also light enough to be considered in the regions of our interest. Before proceeding into the discussion about the second chargino, we should note that the analyses represented in [51] are performed for the winolike chargino that is why the lightest chargino is not allowed to decay into a right-handed stau, even if it is allowed kinematically (i.e. $m_{\tilde{\tau}_{R}} \gtrsim m_{\tilde{\chi}_{1}}^{ \pm}$). When the lightest chargino is formed mostly by winos, then the second chargino is formed mostly by Higgsinos. The processes involving the Higgsino-like chargino yield different results, even though the same processes are being considered. First of all, its production and decays occur through the Yukawa interactions. These interactions are significantly suppressed in the chargino-neutralino production processes, since the Yukawa coupling associated with the up and down quarks are quite small that they are, in general, assumed to be zero in calculations. Apart from its production, we consider some relevant decay processes involving the second chargino without regarding whether it is mostly Higgsino or wino. Figure 12 displays our results for the second chargino

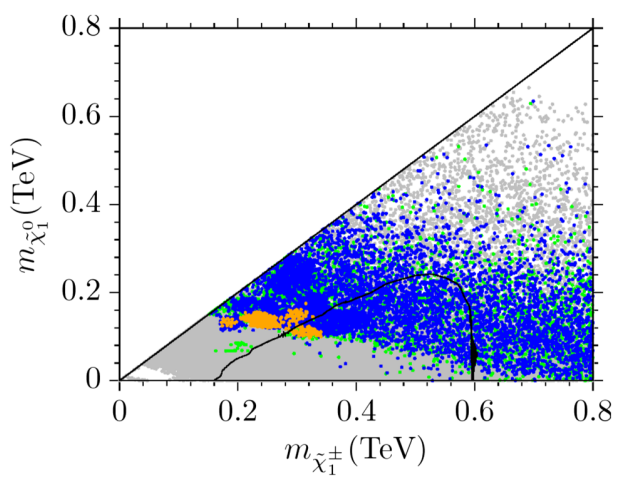

FIG. 11. Results in the $m_{\tilde{\chi}_{1}^{0}}-m_{\tilde{\chi}_{1}^{ \pm}}$plane. The color coding is the same as Fig. 9. The diagonal line indicates the mass degeneracy between the lightest chargino and neutralino. The solid black curve represents the exclusion curve obtained in [52]. 

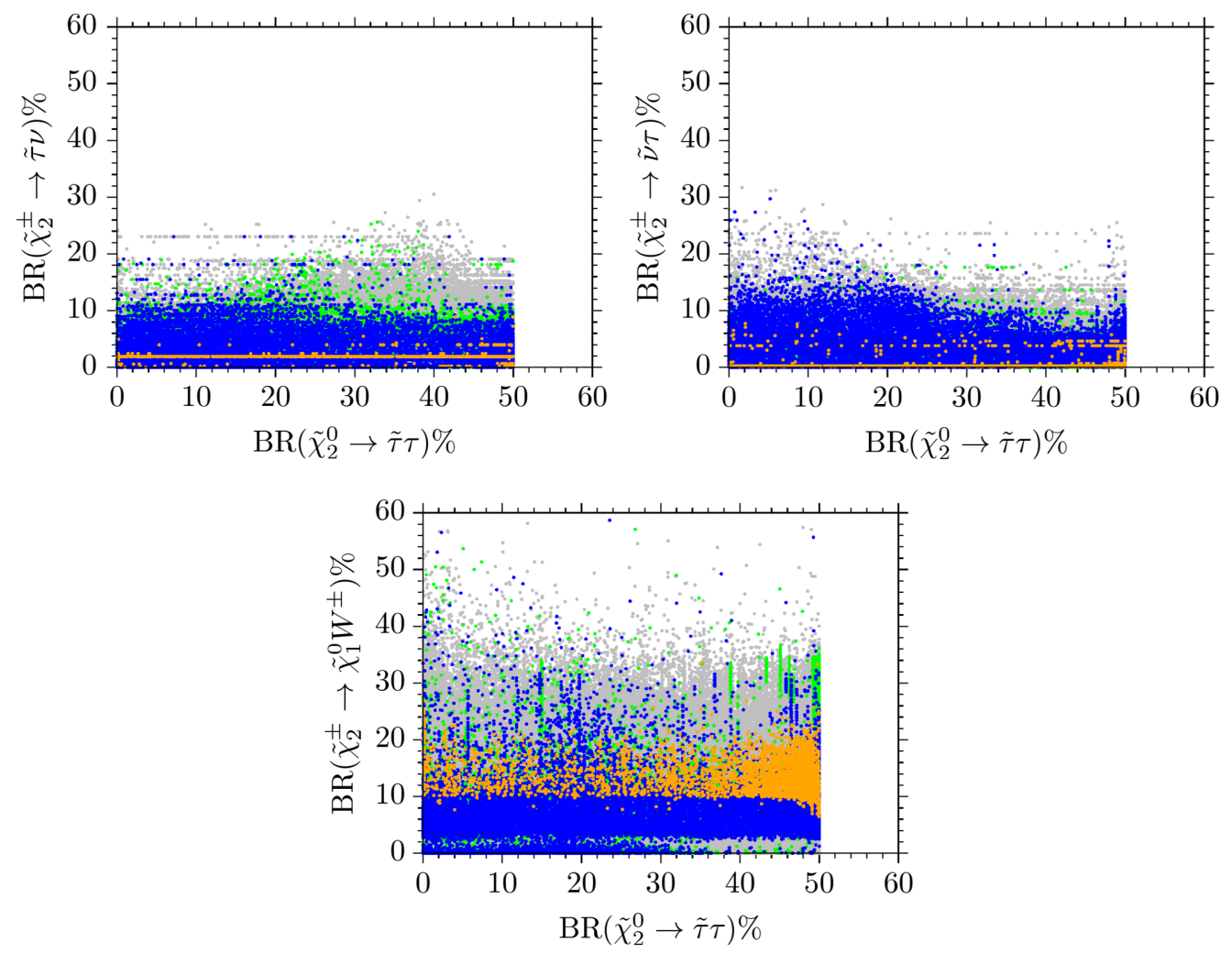

FIG. 12. The second chargino and second neutralino decays with plots in the $\operatorname{BR}\left(\tilde{\chi}_{2}^{ \pm} \rightarrow \tilde{\tau} \nu\right)-\operatorname{BR}\left(\tilde{\chi}_{1}^{0} \rightarrow \tilde{\tau} \tau\right), \operatorname{BR}\left(\tilde{\chi}_{2}^{ \pm} \rightarrow \tilde{\nu} \tau\right)-$ $\operatorname{BR}\left(\tilde{\chi}_{1}^{0} \rightarrow \tilde{\tau} \tau\right)$ and $\operatorname{BR}\left(\tilde{\chi}_{2}^{ \pm} \rightarrow \tilde{\chi}_{1}^{0} W^{ \pm}\right)-\operatorname{BR}\left(\tilde{\chi}_{1}^{0} \rightarrow \tilde{\tau} \tau\right)$ planes. The color coding is the same as Fig. 9.

and second neutralino decays with plots in the $\mathrm{BR}\left(\tilde{\chi}_{2}^{ \pm} \rightarrow \tilde{\tau} \nu\right)-\mathrm{BR}\left(\tilde{\chi}_{2}^{0} \rightarrow \tilde{\tau} \tau\right), \operatorname{BR}\left(\tilde{\chi}_{2}^{ \pm} \rightarrow \tilde{\nu} \tau\right)-\mathrm{BR}\left(\tilde{\chi}_{2}^{0} \rightarrow \tilde{\tau} \tau\right)$ and $\operatorname{BR}\left(\tilde{\chi}_{2}^{ \pm} \rightarrow \tilde{\chi}_{1}^{0} W^{ \pm}\right)-\operatorname{BR}\left(\tilde{\chi}_{2}^{0} \rightarrow \tilde{\tau} \tau\right)$ planes. The color coding is the same as Fig. 9. The $\operatorname{BR}\left(\tilde{\chi}_{2}^{ \pm} \rightarrow \tilde{\tau} \nu\right)-$ $\operatorname{BR}\left(\tilde{\chi}_{1}^{0} \rightarrow \tilde{\tau} \tau\right)$ plane shows that the branching ratio for the $\tilde{\chi}_{2}^{ \pm} \rightarrow \tilde{\tau} \nu$ mode is always less than $30 \%$ (blue), and the orange region compatible with the resolution to the muon $g-2$ problem within $1 \sigma$ restricts the solutions as $\operatorname{BR}\left(\tilde{\chi}_{2}^{ \pm} \rightarrow \tilde{\tau} \nu\right) \lesssim 10 \%$. Similarly the orange region predicts $\operatorname{BR}\left(\tilde{\chi}_{2}^{ \pm} \rightarrow \tilde{\nu} \tau\right) \lesssim 10 \%$ as seen in the $\operatorname{BR}\left(\tilde{\chi}_{2}^{ \pm} \rightarrow \tilde{\nu} \tau\right)-$ $\operatorname{BR}\left(\tilde{\chi}_{1}^{0} \rightarrow \tilde{\tau} \tau\right)$ plane. Such small branching ratios suppress the possible signals when the second chargino decays into the staus (or sneutrinos); thus, the solutions can still be considered viable under the current analyses. Besides the decay modes to staus, the second chargino can also decay into the lightest neutralino along with a $W^{ \pm}$boson. However, the $\operatorname{BR}\left(\tilde{\chi}_{2}^{ \pm} \rightarrow \tilde{\chi}_{1}^{0} W^{ \pm}\right)-\operatorname{BR}\left(\tilde{\chi}_{1}^{0} \rightarrow \tilde{\tau} \tau\right)$ plane shows that the branching ratio for this process can barely reach to $30 \%$. If we applies the condition $\operatorname{BR}\left(\tilde{\chi}_{2}^{ \pm} \rightarrow\right.$ possible final states $) \leq$ $30 \%$, as we did for the lightest chargino, all solutions in orange can satisfy this condition. Note that the second chargino can also decay into the second neutralino along
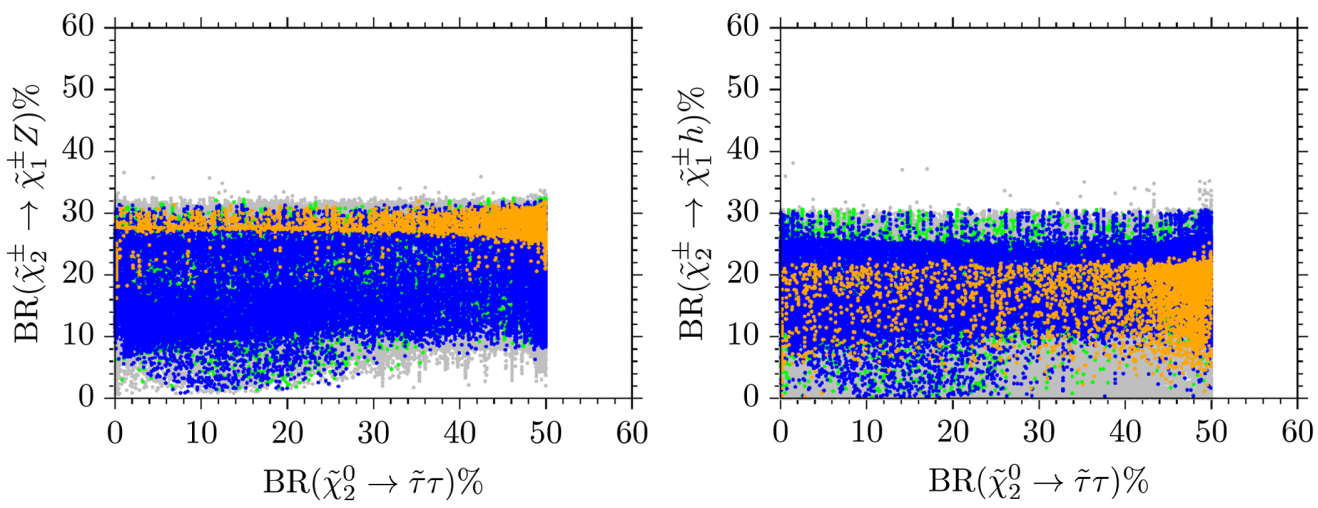

FIG. 13. The second chargino decays into the lightest chargino with plots in the $\operatorname{BR}\left(\tilde{\chi}_{2}^{ \pm} \rightarrow \tilde{\chi}_{1}^{ \pm} Z\right)-\operatorname{BR}\left(\tilde{\chi}_{2}^{0} \rightarrow \tilde{\tau} \tau\right)$ and $\operatorname{BR}\left(\tilde{\chi}_{2}^{ \pm} \rightarrow\right.$ $\left.\tilde{\chi}_{1}^{ \pm} h\right)-\operatorname{BR}\left(\tilde{\chi}_{2}^{0} \rightarrow \tilde{\tau} \tau\right)$ planes, where $h$ stands for the SM like Higgs boson. The color coding is the same as Fig. 9. 
with $W^{ \pm}$boson, however, since this process adds one more branch to the processes considered in this section, its branching ratio rather provides an extra suppression factor.

One can expect the second chargino to exhibit some tendency to decay into the lightest chargino. We present our results for such decay modes in Fig. 13 with plots in the $\operatorname{BR}\left(\tilde{\chi}_{2}^{ \pm} \rightarrow \tilde{\chi}_{1}^{ \pm} Z\right)-\operatorname{BR}\left(\tilde{\chi}_{2}^{0} \rightarrow \tilde{\tau} \tau\right)$ and $\operatorname{BR}\left(\tilde{\chi}_{2}^{ \pm} \rightarrow \tilde{\chi}_{1}^{ \pm} h\right)-$ $\operatorname{BR}\left(\tilde{\chi}_{2}^{0} \rightarrow \tilde{\tau} \tau\right)$ planes, where $h$ stands for the SM like Higgs boson. The color coding is the same as Fig. 9. As seen from the both planes, the decay modes for the second chargino into the lightest chargino can happen along with either a $Z$ boson or the SM like Higgs boson, and the branching ratios for these processes are observed as $\operatorname{BR}\left(\tilde{\chi}_{2}^{ \pm} \rightarrow \tilde{\chi}_{1}^{ \pm} Z\right) \sim 30 \%$, while $\operatorname{BR}\left(\tilde{\chi}_{2}^{ \pm} \rightarrow \tilde{\chi}_{1}^{ \pm} h\right) \lesssim 25 \%$. As a result, despite being light enough, the second chargino can be allowed under the current results from the analyses, since the model under concern predicts rather low branching ratios (and so low cross-sections) for the relevant decay modes considered in the current analyses.

Besides the chargino and neutralino, the mass bounds on the charged sleptons are strongly related to the processes which start with the chargino-neutralino production [54]. Thus, the discussion above can be also followed for the bounds on the slepton masses.

TABLE I. Table of four benchmark points, which exemplify our findings. All points have been chosen as to be consistent with the current experimental constraints except those from the dark matter observations. All masses are given in GeV, and cross-sections in pb.

\begin{tabular}{|c|c|c|c|c|}
\hline & Point 1 & Point 2 & Point 3 & Point 4 \\
\hline$m_{0}$ & 644.2 & 791.2 & 644.5 & 721.3 \\
\hline$m_{H_{d}}$ & 1612 & 1544 & 1668 & 1438 \\
\hline$m_{H_{u}}$ & 2007 & 2359 & 2015 & 2280 \\
\hline$M_{2}$ & -277.5 & -331.9 & -285.9 & -332.7 \\
\hline$M_{3}$ & 1241 & 1458 & 1249 & 1419 \\
\hline$A_{0} / m_{0}$ & -0.71 & 0.28 & -0.77 & 0.26 \\
\hline $\tan \beta$ & 37.6 & 39.7 & 36.8 & 38.9 \\
\hline$m_{h}$ & 124 & 123.6 & 124.7 & 123.8 \\
\hline$m_{H}$ & 1141 & 527.7 & 1226 & 576.8 \\
\hline$m_{A}$ & 929.2 & 531.6 & 1038 & 581 \\
\hline$m_{H^{ \pm}}$ & 933.5 & 538.9 & 1042 & 587.5 \\
\hline$\Delta a_{\mu} \times 10^{10}$ & 25.5 & 21.3 & 24.4 & 24.3 \\
\hline$\Omega h^{2}$ & 0.07 & 0.10 & 0.08 & 0.11 \\
\hline$m_{\tilde{g}}$ & 2726 & 3165 & 2743 & 3083 \\
\hline$m_{\tilde{u}_{L, R}}$ & 2349,2364 & 2728,2754 & 2363,2376 & 2646,2672 \\
\hline$m_{\tilde{t}_{1,2}}$ & 1823,2042 & 2160,2381 & 1832,2058 & 2100, 2329 \\
\hline$m_{\tilde{d}_{L, R}}$ & 2349,2350 & 2728,2727 & 2363,2364 & 2646, 2644 \\
\hline$m_{\tilde{b}_{1,2}}$ & 2016,2184 & 2361,2548 & 2033,2205 & 2309,2502 \\
\hline$m_{\tilde{e}_{L . R}}$ & 686,595 & $854.3,694.4$ & 685,601 & 790,614 \\
\hline$m_{\tilde{\tau}_{1,2}}$ & $180.5,494.7$ & $282.2,527.7$ & $191.8,493.6$ & $254.6,506.1$ \\
\hline$m_{\tilde{\chi}_{1,2}^{0}}$ & $129,234.7$ & $152.1,300$ & $128.3,240.6$ & $145,292.3$ \\
\hline$m_{\tilde{\chi}_{3,4}^{0}}$ & $340.8,368.7$ & $455.5,472.1$ & $343.1,372.5$ & $413.3,437.3$ \\
\hline$m_{\tilde{\chi}_{1,2}^{ \pm}}$ & $235.2,370.9$ & $300.1,474.5$ & $241.1,374.6$ & $292.6,439.1$ \\
\hline $\operatorname{BR}\left(\tilde{\chi}_{2}^{0} \rightarrow \tilde{\tau} \tau\right) \%$ & 48 & 27 & 46 & 22 \\
\hline $\operatorname{BR}\left(\tilde{\chi}_{2}^{0} \rightarrow \tilde{\chi}_{1}^{0} Z\right) \%$ & 3.2 & 43 & 6.9 & 13 \\
\hline $\operatorname{BR}\left(\tilde{\chi}_{1}^{ \pm} \rightarrow \tilde{\nu} \tau\right) \%$ & 44 & 29 & 39 & 28 \\
\hline $\operatorname{BR}\left(\tilde{\chi}_{1}^{ \pm} \rightarrow \tilde{\chi}_{1}^{0} W^{ \pm}\right) \%$ & 12 & 41 & 21 & 14 \\
\hline $\operatorname{BR}\left(\tilde{\chi}_{2}^{ \pm} \rightarrow \tilde{\tau} \nu\right) \%$ & 8.8 & 12 & 8.0 & 12 \\
\hline $\operatorname{BR}\left(\tilde{\chi}_{2}^{ \pm} \rightarrow \tilde{\chi}_{1}^{ \pm} Z\right) \%$ & 31 & 36 & 30 & 28 \\
\hline $\operatorname{BR}\left(\tilde{\chi}_{2}^{ \pm} \rightarrow \tilde{\chi}_{i}^{0} W^{ \pm}\right) \%$ & 51 & 36 & 53 & 38 \\
\hline $\operatorname{BR}\left(\tilde{\tau} \rightarrow \tau \tilde{\chi}_{1}^{0}\right)$ & 100 & 100 & 100 & 100 \\
\hline$\sigma\left(p p \rightarrow \tilde{\chi}_{1}^{ \pm} \tilde{\chi}_{2}^{0}\right)$ & 0.51 & 0.23 & 0.46 & 0.23 \\
\hline$\sigma\left(p p \rightarrow \tilde{\chi}_{2}^{ \pm} \tilde{\chi}_{2}^{0}\right)$ & $9.2 \times 10^{-3}$ & $1.8 \times 10^{-3}$ & $9.0 \times 10^{-3}$ & $1.8 \times 10^{-3}$ \\
\hline
\end{tabular}


Before concluding we present benchmark points which exemplify our findings in Table I. All points have been chosen as to be consistent with the muon $g-2$ and QYU conditions, and the experimental constraints listed in Section III. All masses are given in GeV, and cross-sections in $\mathrm{pb}$. In addition to the conditions and constraints employed in our analyses, we have also provided the dark matter relic density $\left(\Omega h^{2}\right)$ with use of MicrOmegas [55] and the chargino-neutralino production cross-section $(\sigma(p p \rightarrow$ $\left.\tilde{\chi}_{1,2}^{ \pm} \tilde{\chi}_{1}^{0}\right)$ ) by employing MadGraph [56] for $\sqrt{s}=13 \mathrm{TeV}$. All masses are given in $\mathrm{GeV}$, and cross-sections in pb. All points yield light mass spectrum for the electroweak sector including the sleptons, while the hadronic sector rather involves heavy squarks and gluino. Point 1 depicts a solution which predicts large branching ratios as $\operatorname{BR}\left(\tilde{\chi}_{1}^{ \pm} \rightarrow \tilde{\nu} \tau\right)=$ $44 \%$ and $\operatorname{BR}\left(\tilde{\chi}_{2}^{0} \rightarrow \tilde{\tau} \tau\right)=48 \%$. Even though such solutions with the branching ratios greater than $30 \%$ can be considered potentially to be excluded, the following approximation can provide slightly deeper understanding:

$$
\begin{aligned}
& \sigma\left(p p \rightarrow \tilde{\chi}_{i}^{0} \tilde{\chi}_{2}^{0} \rightarrow \tilde{\tau} \tilde{\nu} \tau \tau\right) \\
& \quad \approx \sigma\left(p p \rightarrow \tilde{\chi}_{i}^{ \pm} \tilde{\chi}_{2}^{0}\right) \times \operatorname{BR}\left(\tilde{\chi}_{i}^{ \pm} \rightarrow \tilde{\nu} \tau\right) \times \operatorname{BR}\left(\tilde{\chi}_{2}^{0} \rightarrow \tilde{\tau} \tau\right)
\end{aligned}
$$

where $i=1,2$ stand for the two chargino mass eigenstates. Since the regions of our interest yield both chargino to be light, Eq. (11) can be applied for the two charginos separately.

In this context, Point 1 yields $m_{\tilde{\chi}_{1}^{0}}=129 \mathrm{GeV}, m_{\tilde{\chi}_{1}^{ \pm}}=$ $340.8 \mathrm{GeV}$, and $\sigma\left(p p \rightarrow \tilde{\chi}_{i}^{0} \tilde{\chi}_{2}^{0} \rightarrow \tilde{\tau} \tilde{\nu} \tau\right) \sim 0.1 \mathrm{pb}$. As is expected, it leads to a large cross-section for the possible signal. Even though the sneutrino can manifest itself by contributing to the missing energy resulted from the total signal process, the experimental analyses are capable to trace back to the sneutrino. However, in the mass plane of the lightest neutralino and chargino, the exclusion takes part on such solutions, when $\sigma\left(p p \rightarrow \tilde{\chi}_{i}^{0} \tilde{\chi}_{2}^{0} \rightarrow \tilde{\tau} \tilde{\nu} \tau \tau\right) \sim 1$ (reading from the color legend in Fig. 13 of Ref. [51]). This result makes the signal about ten times weaker, in compared to those analyzed in Ref. [51], against the background processes; thus, the exclusion analyses over such solutions need to be applied with stronger cuts and/or higher luminosity. In this sense, Point 1 can still be considered as a testable solution. Point 2 represents the heaviest chargino solutions bounded by the muon $g-2$ condition, which we can realize within our data, while the Point 3 displays the heaviest SM-like Higgs solutions, and Point 4 is chosen for the solutions predicting very light mass scales for the charginos. The discussion for the weakness of the signal holds also for these points.

The relic density is found slightly lower than the $5 \sigma$ bound provided by the WMAP [45] and the Planck [46] satellites for Points 1 and 3, while Points 2 and 4 are consistent with the current measurements within $5 \sigma$ uncertainty. We also list the second chargino's behavior in its decay patterns. The solutions show that the second chargino mostly tends to decay into the lightest chargino or the neutralino along with the possible SM particles as $\operatorname{BR}\left(\tilde{\chi}_{2}^{ \pm} \rightarrow \tilde{\chi}_{1}^{ \pm} Z\right) \sim 30 \%$, while $\operatorname{BR}\left(\tilde{\chi}_{2}^{ \pm} \rightarrow \tilde{\chi}_{i}^{0} W^{ \pm}\right)$varies from about $30 \%$ to $50 \%$. The production processes for the second chargino yield cross-sections two magnitudes smaller than the first chargino production, and one can expect much weaker signal for the second chargino.

\section{CONCLUSION}

We explore the low-scale implications of $4-2-2$ including the $\mathrm{TeV}$ scale right-handed neutrinos interacting and mixing with the MSSM fields through the IS mechanism, in light of muon $g-2$ resolution and highlight the solutions which are compatible with the QYU condition. We found that the presence of the right-handed neutrinos cause the smuons are rather heavy as $m_{\tilde{\mu}} \gtrsim 800 \mathrm{GeV}$ in order to avoid tachyonic staus at the low scale. In this context, the usual MSSM contributions to muon $g-2$, which are provided from smuon-neutralino loop, is either suppressed or minor. On the other hand, the sneutrinos can be as light as about $100 \mathrm{GeV}$ along with the light charginos of mass $\lesssim 400 \mathrm{GeV}$ can yield so large contributions to muon $g-2$ that the discrepancy between the experiment and the theory can be resolved. Large sneutrino-chargino contributions also requires light charginos, and the muon $g-2$ resolution provide a bound on the lightest chargino mass as $m_{\tilde{\chi}_{1}^{ \pm}} \lesssim 400 \mathrm{GeV}$. Even though the neutralino contribution is rather suppress or minor due to the heavy smuon masses, its mass cannot be heavier than about $200 \mathrm{GeV}$, since the solutions are required to have one of the neutralinos being LSP always. We have also considered such light chargino and neutralino along with light stau $\left(m_{\tilde{\tau}} \gtrsim 200 \mathrm{GeV}\right)$ in the light of the current results which constrain their masses to be larger even beyond the $\mathrm{TeV}$ scale. We have realized that such solutions can still survive under such severe constraints, when the model is constrained from the GUT scale, and they provide testable solutions in near future experiments. However, our discussion needs to be completed with the analyses on the chargino-neutralino production rate. We discuss also the second chargino, since it is light enough to be subjected in the current analyses in the regions of interest in our study. Our results show that the $\operatorname{BR}\left(\tilde{\chi}_{2}^{ \pm} \rightarrow \tilde{\tau} \nu\right.$ or $\left.\tilde{\nu}\right) \lesssim 10 \%$, while $\operatorname{BR}\left(\tilde{\chi}_{2}^{ \pm} \rightarrow \tilde{\chi}_{1}^{0} W^{ \pm}\right) \lesssim 30 \%$. Since the model under concern predicts rather low branching ratios, the second chargino can survive under the current results, despite being light. We also observe the second chargino to exhibit some tendency to decay into the lightest chargino, which turns to be the first part of the discussion about the status of the lightest chargino in the LHC. We also considered the mass spectrum for other supersymmetric particles. The both stop mass eigenstates are found more or less degenerate in mass as $m_{\tilde{t}_{1}} \sim m_{\tilde{t}_{2}} \gtrsim 1.5 \mathrm{TeV}$ in the region where the muon $g-2$ can be accommodated. The gluino mass lies in a range $\sim[2.5-3.5] \mathrm{TeV}$, which will be tested in near future 
experiments. The $C P$-odd Higgs boson is also found relatively light as $m_{A} \lesssim 1.2 \mathrm{TeV}$.

In addition, the model predicts relatively light Higgsinos $(\mu \lesssim 700 \mathrm{GeV})$; and hence the second chargino mass is also light enough $(\lesssim 700 \mathrm{GeV})$ to contribute to muon $g-2$. Despite the Higgsino mixing in the lightest neutralino and chargino is limited, the light Higgsinos are interesting from the naturalness point of view, since such solutions of the light Higgsinos need to be fine-tuned much less than the other solutions. We found that such solutions can be also compatible with the QYU, since $\Delta_{\mathrm{EW}}$ can be as low as about 100. The acceptable fine-tuning can also have a strong impact on the Yukawa couplings in terms of their ratios, and this impact also shapes the fundamental parameter space of QYU, since it is rather related to the corrections in the Yukawa couplings. In the regions with acceptable fine-tuning and compatible with muon $g-2$ resolution and the QYU condition, the ratios among the Yukawa couplings can be summarized as $1.8 \lesssim y_{t} / y_{b} \lesssim 2.6$, $y_{\tau} / y_{b} \sim 1.3$. In addition, even though the right-handed neutrino Yukawa coupling can be varied freely, the solutions restrict its range as $0.8 \lesssim y_{\nu} / y_{b} \lesssim 1.7$.

\section{ACKNOWLEDGMENTS}

We would like to thank Zerrin Kırca, Büşra Niş and Ali Çiçi for discussions and complementary suggestions. This work is supported by the Scientific and Technological Research Council of Turkey (TUBITAK) Grant No. MFAG-114F461. Part of numerical calculations reported in this paper were performed at the National Academic Network and Information Center (ULAKBIM) of TUBITAK, High Performance anf Grid Computing Center (TRUBA Resources).
[1] R. Wendell et al. (Super-Kamiokande Collaboration), Phys. Rev. D 81, 092004 (2010).

[2] B. Ananthanarayan, G. Lazarides, and Q. Shafi, Phys. Rev. D 44, 1613 (1991); Phys. Lett. B 300, 245 (1993); Q. Shafi and B. Ananthanarayan, in Trieste HEP Cosmology, Vol. 1 (Bartol Research Institute, Newark, NJ, 1991), p. 233; J. C. Pati and A. Salam, Phys. Rev. D 10, 275 (1974).

[3] J. C. Pati and A. Salam, Phys. Rev. D 10, 275 (1974).

[4] I. Gogoladze, R. Khalid, S. Raza, and Q. Shafi, J. High Energy Phys. 12 (2010) 055; D. M. Pierce, J. A. Bagger, K. T. Matchev, and R. j. Zhang, Nucl. Phys. B491, 3 (1997).

[5] P. Langacker, Phys. Rep. 72, 185 (1981).

[6] See, for instance, E. Witten, Phys. Lett. B 91, 81 (1980); S. M. Barr, Phys. Rev. D 21, 1424 (1980); Y. Nomura and T. Yanagida, Phys. Rev. D 59, 017303 (1998); M. Frigerio, P. Hosteins, S. Lavignac, and A. Romanino, Nucl. Phys. B806, 84 (2009); S. M. Barr, Phys. Rev. D 76, 105024 (2007); M. Malinsky, Phys. Rev. D 77, 055016 (2008); M. Heinze and M. Malinsky, Phys. Rev. D 83, 035018 (2011); K. S. Babu, B. Bajc, and Z. Tavartkiladze, Phys. Rev. D 86, 075005 (2012) and references therein.

[7] K. S. Babu and R. N. Mohapatra, Phys. Rev. Lett. 70, 2845 (1993).

[8] For a brief review, see A. S. Joshipura and K. M. Patel, Phys. Rev. D 86, 035019 (2012) and references therein.

[9] M. E. Gomez, G. Lazarides, and C. Pallis, Nucl. Phys. B638, 165 (2002); M. E. Gomez, G. Lazarides, and C. Pallis, Phys. Rev. D 67, 097701 (2003); C. Pallis and M. E. Gomez, arXiv:hep-ph/0303098;

[10] I. Gogoladze, R. Khalid, and Q. Shafi, Phys. Rev. D 79, 115004 (2009).

[11] S. Raza, Q. Shafi, and C. S. Ün, Phys. Rev. D 92, 055010 (2015).

[12] S. Dar, I. Gogoladze, Q. Shafi, and C. S. Un, Phys. Rev. D 84, 085015 (2011); Q. Shafi, Ş. H. Tanyıldızı, and C. S. Un, Nucl.
Phys. B900, 400 (2015); Y. Hiçyılmaz, M. Ceylan, A. Altas, L. Solmaz, and C. S. Un, Phys. Rev. D 94, 095001 (2016).

[13] B. Bajc, A. Melfo, G. Senjanovic, and F. Vissani, Phys. Rev. D 70, 035007 (2004); S. Bertolini, M. Frigerio, and M. Malinsky, Phys. Rev. D 70, 095002 (2004); B. Dutta, Y. Mimura, and R. N. Mohapatra, Phys. Rev. D 69, 115014 (2004); T. Fukuyama, A. Ilakovac, T. Kikuchi, S. Meljanac, and N. Okada, J. Math. Phys. (N.Y.) 46, 033505 (2005).

[14] S. Antusch, S. F. King, and M. Spinrath, Phys. Rev. D 89, 055027 (2014); S. Antusch, L. Calibbi, V. Maurer, M. Monaco, and M. Spinrath, Phys. Rev. D 85, 035025 (2012); S. Antusch and M. Spinrath, Phys. Rev. D 79, 095004 (2009); S. Trine, S. Westhoff, and S. Wiesenfeldt, J. High Energy Phys. 08 (2009) 002.

[15] A. Hebbar, Q. Shafi, and C. S. Un, Phys. Rev. D 95, 115026 (2017) and references therein.

[16] C. Coriano, L. Delle Rose, and C. Marzo, Nucl. Part. Phys. Proc. 265-266, 311 (2015); S. Khalil and H. Okada, Prog. Theor. Phys. Suppl. 180, 35 (2009); M. Abbas and S. Khalil, J. High Energy Phys. 04 (2008) 056, and references therein.

[17] See, for instance, S. Khalil, Phys. Rev. D 82, 077702 (2010); A. Masiero, S. K. Vempati, and O. Vives, Nucl. Phys. B, Proc. Suppl. 137, 156 (2004).

[18] G. W. Bennett et al. (Muon g-2 Collaboration), Phys. Rev. D 73, 072003 (2006); G. W. Bennett et al. (Muon (g-2) Collaboration), Phys. Rev. D 80, 052008 (2009).

[19] M. Davier, A. Hoecker, B. Malaescu, and Z. Zhang, Eur. Phys. J. C 71, 1515 (2011); 72, 1874 (2012); K. Hagiwara, R. Liao, A. D. Martin, D. Nomura, and T. Teubner, J. Phys. G 38, 085003 (2011).

[20] K. S. Babu, I. Gogoladze, Q. Shafi, and C. S. Ün, Phys. Rev. D 90, 116002 (2014); M. A. Ajaib, I. Gogoladze, Q. Shafi, and C. S. Ün, J. High Energy Phys. 05 (2014) 079; I. Gogoladze, F. Nasir, Q. Shafi, and C. S. Un, Phys. Rev. D 90, 035008 (2014); I. Gogoladze, Q. Shafi, and C. S. Ün, 
Phys. Rev. D 92, 115014 (2015); B. P. Padley, K. Sinha, and K. Wang, Phys. Rev. D 92, 055025 (2015), and references therein.

[21] E. J. Chun, V. S. Mummidi, and S. K. Vempati, Phys. Lett. B 736, 470 (2014); I. Gogoladze, B. He, A. Mustafayev, S. Raza, and Q. Shafi, J. High Energy Phys. 05 (2014) 078, and references therein.

[22] See, for instance, I. Gogoladze, B. He, and Q. Shafi, Phys. Lett. B 718, 1008 (2013).

[23] J. L. Lopez, D. V. Nanopoulos, and X. Wang, Phys. Rev. D 49, 366 (1994); U. Chattopadhyay and P. Nath, Phys. Rev. D 53, 1648 (1996); T. Moroi, Phys. Rev. D 53, 6565 (1996); 56, 4424 (1997); S. P. Martin and J. D. Wells, Phys. Rev. D 64, 035003 (2001); G. F. Giudice, P. Paradisi, A. Strumia, and A. Strumia, J. High Energy Phys. 10 (2012) 186.

[24] S. Khalil and C. S. Un, Phys. Lett. B 763, 164 (2016).

[25] I. Gogoladze, B. He, A. Mustafayev, S. Raza, and Q. Shafi, J. High Energy Phys. 05 (2014) 078.

[26] See, for instance, H. Baer, V. Barger, P. Huang, D. Mickelson, A. Mustafayev, and X. Tata, Phys. Rev. D 87, 035017 (2013); D. A. Demir and C. S. Ün, Phys. Rev. D 90, 095015 (2014); A. Cici, Z. Kirca, and C. S. Un, Eur. Phys. J. C 78, 60 (2018) and references therein.

[27] W. Porod, Comput. Phys. Commun. 153, 275 (2003); W. Porod and F. Staub, Comput. Phys. Commun. 183, 2458 (2012).

[28] F. Staub, arXiv:0806.0538.

[29] J. Hisano, H. Murayama, and T. Yanagida, Nucl. Phys. B402, 46 (1993); J. L. Chkareuli and I. G. Gogoladze, Phys. Rev. D 58, 055011 (1998).

[30] T.E. W. Group (CDF and D0 Collaborations), arXiv: 0903.2503.

[31] I. Gogoladze, R. Khalid, S. Raza, and Q. Shafi, J. High Energy Phys. 06 (2011) 117.

[32] I. Gogoladze, Q. Shafi, and C. S. Un, J. High Energy Phys. 08 (2012) 028; M. Adeel Ajaib, I. Gogoladze, Q. Shafi, and C. S. Un, J. High Energy Phys. 07 (2013) 139.

[33] B. O'Leary, W. Porod, and F. Staub, J. High Energy Phys. 05 (2012) 042.

[34] See, incomplete list of references, L. J. Hall, R. Rattazzi, and U. Sarid, Phys. Rev. D 50, 7048 (1994); B. Ananthanarayan, Q. Shafi, and X. Wang, Phys. Rev. D 50, 5980 (1994); R. Rattazzi and U. Sarid, Phys. Rev. D 53, 1553 (1996); T. Blazek, M. Carena, S. Raby, and C. Wagner, Phys. Rev. D 56, 6919 (1997); J. L. Chkareuli and I. G. Gogoladze, Phys. Rev. D 58, 055011 (1998); T. Blazek, S. Raby, and K. Tobe, Phys. Rev. D 62, 055001 (2000); H. Baer, M. Brhlik, M. Diaz, J. Ferrandis, P. Mercadante, P. Quintana, and X. Tata, Phys. Rev. D 63, 015007 (2000); C. Balazs and R. Dermisek, J. High Energy Phys. 06 (2003) 024; U. Chattopadhyay, A. Corsetti, and P. Nath, Phys. Rev. D 66, 035003 (2002); T. Blazek, R. Dermisek, and S. Raby, Phys. Rev. Lett. 88, 111804 (2002); M. Gomez, T. Ibrahim, P. Nath, and S. Skadhauge, Phys. Rev. D 72, 095008 (2005); K. Tobe and J. D. Wells, Nucl. Phys. B663, 123 (2003); I. Gogoladze, Y. Mimura, and S. Nandi, Phys. Lett. B 562, 307 (2003); W. Altmannshofer, D. Guadagnoli, S. Raby, and D. M. Straub, Phys. Lett. B 668, 385 (2008); S. Antusch and M. Spinrath, Phys. Rev. D 78, 075020 (2008); H. Baer, S. Kraml, and S. Sekmen, J. High Energy Phys. 09 (2009) 005; S. Antusch and M. Spinrath, Phys. Rev. D 79, 095004 (2009); K. Choi, D. Guadagnoli,
S. H. Im, and C. B. Park, J. High Energy Phys. 10 (2010) 025; M. Badziak, M. Olechowski, and S. Pokorski, J. High Energy Phys. 08 (2011) 147; S. Antusch, L. Calibbi, V. Maurer, M. Monaco, and M. Spinrath, Phys. Rev. D 85, 035025 (2012); J. S. Gainer, R. Huo, and C. E. M. Wagner, J. High Energy Phys. 03 (2012) 097; H. Baer, S. Raza, and Q. Shafi, Phys. Lett. B 712, 250 (2012); I. Gogoladze, Q. Shafi, and C. S. Un, J. High Energy Phys. 07 (2012) 055; M. Badziak, Mod. Phys. Lett. A 27, 1230020 (2012); G. Elor, L. J. Hall, D. Pinner, and J. T. Ruderman, J. High Energy Phys. 10 (2012) 111; I. Gogoladze, Q. Shafi, and C. S. Un, Phys. Lett. B 704, 201 (2011); I. Gogoladze, Q. Shafi, and C. S. Un, J. High Energy Phys. 08 (2012) 028; M. A. Ajaib, I. Gogoladze, and Q. Shafi, arXiv:1307.4882.M. A. Ajaib, I. Gogoladze, Q. Shafi, and C. S. Un, J. High Energy Phys. 07 (2013) 139; M. A. Ajaib, I. Gogoladze, Q. Shafi, and C. S. Un, arXiv:1308.4652.

[35] L. E. Ibanez and G. G. Ross, Phys. Lett. 110B, 215 (1982); K. Inoue, A. Kakuto, H. Komatsu, and S. Takeshita, Prog. Theor. Phys. 68, 927 (1982); L. E. Ibanez, Phys. Lett. 118B, 73 (1982); J. R. Ellis, D. V. Nanopoulos, and K. Tamvakis, Phys. Lett. 121B, 123 (1983); L. Alvarez-Gaume, J. Polchinski, and M. B. Wise, Nucl. Phys. B221, 495 (1983).

[36] K. Nakamura et al. (Particle Data Group Collaboration), J. Phys. G 37, 075021 (2010).

[37] See, for instance, H. Baer, I. Gogoladze, A. Mustafayev, S. Raza, and Q. Shafi, J. High Energy Phys. 03 (2012) 047; T. Li, D. V. Nanopoulos, S. Raza, and X. C. Wang, J. High Energy Phys. 08 (2014) 128.

[38] G. Belanger, F. Boudjema, A. Pukhov, and R. K. Singh, J. High Energy Phys. 11 (2009) 026; H. Baer, S. Kraml, S. Sekmen, and H. Summy, J. High Energy Phys. 03 (2008) 056.

[39] K. A. Olive et al. (Particle Data Group Collaboration), Chin. Phys. C 38, 090001 (2014).

[40] R. Aaij et al. (LHCb Collaboration), Phys. Rev. Lett. 110, 021801 (2013).

[41] Y. Amhis et al. (Heavy Flavor Averaging Group Collaboration), arXiv:1207.1158.

[42] G. Aad et al. (ATLAS Collaboration), Phys. Lett. B 716, 1 (2012); S. Chatrchyan et al. (CMS Collaboration), J. High Energy Phys. 06 (2013) 081.

[43] The ATLAS collaboration, Report No. ATLAS-CONF2015-067.

[44] J. Beringer et al. (Particle Data Group), Phys. Rev. D 86, 010001 (2012).

[45] G. Hinshaw et al. (WMAP Collaboration), Astrophys. J. Suppl. Ser. 208, 19 (2013).

[46] P. A. R. Ade et al. (Planck Collaboration), Astron. Astrophys. 594, A13 (2016).

[47] I. Gogoladze, M. U. Rehman, and Q. Shafi, Phys. Rev. D 80, 105002 (2009).

[48] A. M. Sirunyan et al. (CMS Collaboration), Phys. Rev. D 96, 032003 (2017); The ATLAS Collaboration (ATLAS Collaboration), Repor No. ATLAS-CONF-2017-021.

[49] H. Baer, V. Barger, J. S. Gainer, P. Huang, M. Savoy, D. Sengupta, and X. Tata, Eur. Phys. J. C 77, 499 (2017); H. Baer, V. Barger, J. S. Gainer, P. Huang, M. Savoy, H. Serce, and X. Tata, Phys. Lett. B 774, 451 (2017), and references therein.

[50] V. Khachatryan et al. (CMS Collaboration), J. High Energy Phys. 10 (2014) 160. 
[51] CMS Collaboration (CMS Collaboration), Report No. CMS-PAS-SUS-16-039.

[52] A. M. Sirunyan et al. (CMS Collaboration), J. High Energy Phys. 11 (2017) 029; CMS Collaboration (CMS Collaboration), Report No. CMS-PAS-SUS-16-034.

[53] A. Choudhury and S. Mondal, Phys. Rev. D 94, 055024 (2016).
[54] See, for instance, A. Robichaud-Véronneau (ATLAS Collaboration), Nucl. Part. Phys. Proc. 273-275, 570 (2016).

[55] G. Belanger, F. Boudjema, A. Pukhov, and A. Semenov, Comput. Phys. Commun. 176, 367 (2007); 185, 960 (2014).

[56] J. Alwall, M. Herquet, F. Maltoni, O. Mattelaer, and T. Stelzer, J. High Energy Phys. 06 (2011) 128. 